\title{
HOW TO PROVE THE DISCRETE RELIABILITY FOR NONCONFORMING FINITE ELEMENT METHODS*
}

\author{
Carsten Carstensen and Sophie Puttkammer \\ Department of Mathematics, Humboldt-Universität zu Berlin, Unter den Linden 6, \\ 10099 Berlin, Germany \\ Email: cc@math.hu-berlin.de, puttkams@math.hu-berlin.de
}

\begin{abstract}
Optimal convergence rates of adaptive finite element methods are well understood in terms of the axioms of adaptivity. One key ingredient is the discrete reliability of a residualbased a posteriori error estimator, which controls the error of two discrete finite element solutions based on two nested triangulations. In the error analysis of nonconforming finite element methods, like the Crouzeix-Raviart or Morley finite element schemes, the difference of the piecewise derivatives of discontinuous approximations to the distributional gradients of global Sobolev functions plays a dominant role and is the object of this paper. The nonconforming interpolation operator, which comes natural with the definition of the aforementioned nonconforming finite element in the sense of Ciarlet, allows for stability and approximation properties that enable direct proofs of the reliability for the residual that monitors the equilibrium condition. The novel approach of this paper is the suggestion of a right-inverse of this interpolation operator in conforming piecewise polynomials to design a nonconforming approximation of a given coarse-grid approximation on a refined triangulation. The results of this paper allow for simple proofs of the discrete reliability in any space dimension and multiply connected domains on general shape-regular triangulations beyond newest-vertex bisection of simplices. Particular attention is on optimal constants in some standard discrete estimates listed in the appendices.
\end{abstract}

Mathematics subject classification: $65 \mathrm{~N} 30$.

Key words: Discrete reliability, Nonconforming finite element method, Conforming companion, Morley, Crouzeix-Raviart, Explicit constants, Axioms of adaptivity.

\section{Introduction}

\subsection{Motivation}

The nonconforming finite element schemes are a subtile but important part of the finite element practice not exclusively in computational fluid dynamics [1-3], but also with benefits for guaranteed lower bounds of eigenvalues [5,9], lower bounds for energies e.g. in the obstacle problem [13], or guaranteed convergence for a convex energy density despite the presence of the Lavrentiev phenomenon [23]. Prominent examples are Crouzeix-Raviart [17] and Morley [22] finite elements illustrated in Fig. 1.1.a and d.

The discrete reliability is one key-property in the overall analysis of optimal convergence rates in adaptive mesh-refining algorithms and one axiom in [4,15]. Its proof is a challenge in the nonconforming setting since even given an admissible refinement $\widehat{\mathcal{T}}$ of an regular triangulation $\mathcal{T}$ the associated finite element spaces are non-nested $V(\widehat{\mathcal{T}}) \not \subset V(\mathcal{T})$.

\footnotetext{
* Received August 13, 2018 / Revised version received April 1, 2019 / Accepted August 30, 2019 /

Published online January 14, 2020 /
} 


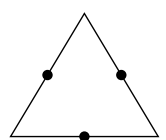

(a) Crouzeix-Raviart

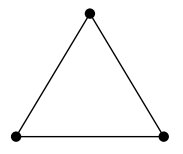

(b) $P_{1}$

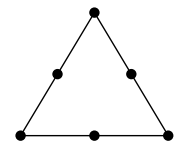

(c) $P_{2}$

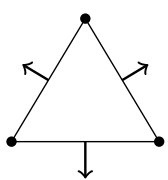

(d) Morley

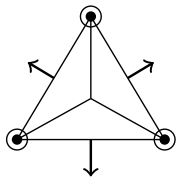

(e) $\mathrm{HCT}$

Fig. 1.1. Mnemonic diagrams of the finite elements in 2D.

\subsection{Methodology}

The authors see three different arguments (i)-(iii) to circumvent the non-nestedness of the nonconforming schemes in the literature,

(i) appropriate mesh-refining,

(ii) discrete Helmholtz decomposition,

(iii) conforming companions.

For Crouzeix-Raviart finite elements see Theorem 2.1 in [24] for (ii). The restriction to simplyconnected domains and dimension $n=2$ from (ii) is circumvented in [7] for Crouzeix-Raviart using intermediate triangulations (i) and an associated discrete quasi-interpolation. For the Morley finite element analysis see Lemma 5.5 in [20] for (i) and Theorem 4.1 in [6] for (ii). This paper presents (iii) and its application for more general and refined results to prove discrete reliability. This general domain independent principle shall serve as a guideline for the many nonconforming methods in the rich literature. Often a discrete Helmholtz decomposition is not available, however the construction of a conforming companion although allows to compute guaranteed upper error bounds. Therefore, it seems intuitive to use this operator for the proof of discrete reliability as outlined in this paper.

\subsection{Model Problems}

For better intuition the reader may have the following model problems in mind. Given a polyhedral Lipschitz domain $\Omega \subset \mathbb{R}^{n}$ and a right-hand side $f \in L^{2}(\Omega)$, for a second-order problem consider the Poisson Model Problem, find $u \in H^{1}(\Omega)$ with

$$
\Delta u=f \text { in } \Omega \quad \text { and } \quad u=0 \text { along } \partial \Omega,
$$

where the weak formulation seeks $u \in H_{0}^{1}(\Omega)$ such that

$$
\int_{\Omega} \nabla u \cdot \nabla v \mathrm{~d} x=\int_{\Omega} f v \mathrm{~d} x \quad \text { for all } v \in H_{0}^{1}(\Omega) .
$$

The discrete version of this energy scalar product reads

$$
a_{h}\left(u_{h}, v_{h}\right):=\int_{\Omega} \nabla_{\mathrm{NC}} u_{h} \cdot \nabla_{\mathrm{NC}} v_{h} \mathrm{~d} x \quad \text { for all } u_{h}, v_{h} \in H^{1}(\Omega)+V(\mathcal{T})+V(\widehat{\mathcal{T}}),
$$

where a possible choice for the nonconforming finite element space $V(\mathcal{T})$ is the Crouzeix-Raviart space $C R_{0}^{1}(\mathcal{T})$. A simple fourth-order elliptic problem is the biharmonic equation, which seeks $u \in H^{2}(\Omega)$ with

$$
\Delta^{2} u=f \text { in } \Omega \quad \text { and } \quad u=\frac{\partial u}{\partial \nu}=0 \text { along } \partial \Omega .
$$


The corresponding weak formulation seeks $u \in H_{0}^{2}(\Omega)$ such that

$$
\int_{\Omega} D^{2} u: D^{2} v \mathrm{~d} x=\int_{\Omega} f v \mathrm{~d} x \quad \text { for all } v \in H_{0}^{2}(\Omega) .
$$

The discrete version of the associated energy scalar product reads

$$
a_{h}\left(u_{h}, v_{h}\right):=\int_{\Omega} D_{\mathrm{NC}}^{2} u_{h}: D_{\mathrm{NC}}^{2} v_{h} \mathrm{~d} x \quad \text { for all } u_{h}, v_{h} \in H^{2}(\Omega)+V(\mathcal{T})+V(\widehat{\mathcal{T}})
$$

and a possible choice for the nonconforming finite element space $V(\mathcal{T})$ in the biharmonic setting is the Morley finite element space $M(\mathcal{T})$. In both cases the discrete problem seeks $u_{h} \in V(\mathcal{T})$ such that

$$
a_{h}\left(u_{h}, v_{h}\right)=\int_{\Omega} f v_{h} \mathrm{~d} x \quad \text { for all } v_{h} \in V(\mathcal{T})
$$

\subsection{Results}

Given a regular triangulation $\mathcal{T}$ and its admissible refinement $\widehat{\mathcal{T}}$ with the finite element spaces $V(\mathcal{T})$ (resp. $V(\widehat{\mathcal{T}}))$ and the discrete solutions $u_{h}$ (resp. $\widehat{u}_{h}$ ), the abstract section shows the discrete reliability

$$
\left\|\widehat{u}_{h}-u_{h}\right\|_{h}^{2} \leq \Lambda_{d r e l}^{2} \sum_{T \in \mathcal{R}} \eta^{2}(T) .
$$

Here and throughout this paper, $\eta(T)$ is an error estimator contribution, the discrete norm $\|\cdot\|_{h}$ is induced by a scalar product $a_{h}$ on $V(\mathcal{T})+V(\widehat{\mathcal{T}})$, and $\mathcal{R}:=\{K \in \mathcal{T}: \exists T \in \mathcal{T} \backslash$ $\widehat{\mathcal{T}}$ with $\operatorname{dist}(K, T)=0\}$ is the set $\mathcal{T} \backslash \widehat{\mathcal{T}}$ of coarse but not fine simplices plus one layer of coarse simplices around. The point is that the universal constant $\Lambda_{\text {drel }} \lesssim 1$ solely depends on the shape-regularity of the triangulation $\mathcal{T}$, but neither on levels nor on mesh-sizes. Four abstract conditions $(\mathrm{C} 1)-(\mathrm{C} 4)$ in Section 3.3 below imply the existence of an approximation $\widehat{u}_{h}^{*} \in V(\widehat{\mathcal{T}})$ such that

$$
\frac{2}{1+\sqrt{2}}\left\|\widehat{u}_{h}-u_{h}\right\|_{h} \leq\left\|\widehat{u}_{h}^{*}-u_{h}\right\|_{h}+\Lambda_{1}\left\|h_{\mathcal{T}}^{m} f\right\|_{L^{2}(\mathcal{T} \backslash \widehat{\mathcal{T}})} .
$$

The additional conditions (C5)-(C7) in Section 3.5 below result in

$$
\left\|\widehat{u}_{h}^{*}-u_{h}\right\|_{h}^{2} \leq \Lambda_{2}^{2} \sum_{T \in \mathcal{R}} h_{T} \sum_{F \in \mathcal{F}(T)}\left\|\left[D^{m} u_{h}\right]_{F} \times \nu_{F}\right\|_{L^{2}(F)}^{2} .
$$

Throughout this paper, the piecewise constant function $\left.h_{\mathcal{T}}\right|_{T}=h_{T}=\operatorname{diam}(T)$ is the diameter of the simplex $T \in \mathcal{T} ; \mathcal{F}(T)$ is the set of sides (edges for $n=2$ or faces for $n=3$ ) of $T$ with the tangential jumps $[v]_{F} \times \nu_{F}$ along sides $F$, and $\|\cdot\|_{L^{2}(\mathcal{T} \backslash \widehat{\mathcal{T}})}^{2}:=\sum_{T \in \mathcal{T} \backslash \widehat{\mathcal{T}}}\|\cdot\|_{L^{2}(T)}^{2}$ is the sum of the $L^{2}$-norms on the coarse but not fine simplices. Section 2 summarises the necessary notation. The combination of (1.3)-(1.4) proves (dRel) with the estimator

$$
\eta^{2}(T):=h_{T}^{2 m}\|f\|_{L^{2}(T)}^{2}+h_{T} \sum_{F \in \mathcal{F}(T)}\left\|\left[D^{m} u_{h}\right]_{F} \times \nu_{F}\right\|_{L^{2}(F)}^{2}
$$

for any simplex $T \in \mathcal{T}$ and $\Lambda_{\text {drel }} \leq\left(1+2^{-1 / 2}\right) \max \left\{\Lambda_{1}, \Lambda_{2}\right\}$. The second task of this paper is to sharpen this result; a modification of the companion operator behind $\widehat{u}_{h}^{*}$ allows the proof of (1.4) and thereby (dRel) with $\mathcal{T} \backslash \widehat{\mathcal{T}}$ replacing $\mathcal{R}$. 


\subsection{Outline}

The remaining parts of this paper are organized as follows. Section 2 simply recalls the standard notation and characterizes a finite patch configuration condition for the admissible triangulations (A2) guaranteed for adaptive mesh refining by newest-vertex bisection. The purpose of Section 3 is an overview over the residual-based error analysis written in an abstract format to be accessible for non-experts and to describe the state of the art and the design of the conforming companion in a language with minimal technicalities. The presented abstract conditions (C1) $-(\mathrm{C} 7)$ imply (1.3)-(1.4) and so (dRel). Section 4 (resp. Section 5) on applications starts with the definition of the Crouzeix-Raviart (resp. Morley) finite element scheme and gives the proof of $(\mathrm{C} 1)-(\mathrm{C} 7)$ to answer the question: How do we prove the discrete reliability for nonconforming finite element schemes? In Section $4, u_{h}, V(\mathcal{T}), I_{h}$ etc. from the general analysis are replaced by $u_{C R}, C R_{0}^{1}(\mathcal{T}), I_{\mathrm{NC}}$, and in Section 5 by $u_{M}, M(\mathcal{T}), I_{M}$ etc. Section 6 introduces a modified companion operator for both examples and proves that indeed $\mathcal{T} \backslash \widehat{\mathcal{T}}$ replacing $\mathcal{R}$ is sufficient in (1.4). The appendices highlight a few discrete inequalities with sharp explicit constants utilized throughout the paper to compute $\Lambda_{d r e l}$.

Standard notation on Lebesgue and Sobolev spaces applies throughout this paper; $H^{m}(T)$ abbreviates $H^{m}(\operatorname{int}(T))$ for a compact set $T$ with non-void interior $\operatorname{int}(T)$. Furthermore, $a \lesssim b$ abbreviates $a \leq C b$ with a generic constant $C$ independent of the meshsize $h_{\mathcal{T}}$, while $a \approx b$ stands for $a \lesssim b \lesssim a$.

\section{Notation}

Regular triangulation. Given a regular triangulation $\mathcal{T}$ of a bounded polyhedral Lipschitz domain $\Omega \subset \mathbb{R}^{n}$ into simplices in the sense of Ciarlet [1-3], let $\mathcal{F}($ resp. $\mathcal{F}(\Omega)$ or $\mathcal{F}(\partial \Omega)$ ) denote the set of all (resp. interior or boundary) sides and let $\mathcal{N}(\operatorname{resp} . \mathcal{N}(\Omega)$ or $\mathcal{N}(\partial \Omega)$ ) denote the set of all (resp. interior or boundary) vertices in $\mathcal{T}$. For any simplex $T \in \mathcal{T}$, the set of its vertices reads $\mathcal{N}(T)$ and the set of its sides reads $\mathcal{F}(T)$. The intersection $T_{1} \cap T_{2}$ of two distinct, nondisjoint simplices $T_{1}$ and $T_{2}$ in $\mathcal{T}$ is the shared sub-simplex $\operatorname{conv}\left\{\mathcal{N}\left(T_{1}\right) \cap \mathcal{N}\left(T_{2}\right)\right\}=\partial T_{1} \cap \partial T_{2}$ of their shared vertices.

Given a side $F \in \mathcal{F}$, the side-patch $\omega_{F}:=\operatorname{int}\left(\bigcup_{T \in \mathcal{T}(F)} T\right)$ is the interior of the union $\bigcup \mathcal{T}(F)$ of the set $\mathcal{T}(F):=\{T \in \mathcal{T}: F \in \mathcal{F}(T)\}$ of all simplices with side $F$. Given a vertex $z \in \mathcal{N}$, the nodal patch $\omega_{z}:=\operatorname{int}\left(\bigcup_{T \in \mathcal{T}(z)} T\right)$ is the interior of the union $\bigcup \mathcal{T}(z)$ of the set $\mathcal{T}(z):=\{T \in \mathcal{T}: z \in \mathcal{N}(T)\}$ of all simplices with vertex $z$. For any simplex $T \in \mathcal{T}$ the set $\mathcal{T}(\Omega(T)):=\{K \in \mathcal{T}: \operatorname{dist}(T, K)=0\}$ of simplices in $\mathcal{T}$ near $T$ has cardinality $|\mathcal{T}(\Omega(T))|$ and covers the closure of $\Omega(T):=\operatorname{int}\left(\bigcup_{T \in \mathcal{T}(\Omega(T))} T\right)=\operatorname{int}\left(\bigcup_{z \in \mathcal{N}(T)} \bigcup \mathcal{T}(z)\right)$.

Admissible triangulation. Throughout this paper, $\mathcal{T}$ is computed by successive admissible mesh-refinements of a regular initial triangulation $\mathcal{T}_{0}$. The set $\mathbb{T}$ of admissible triangulations of all those triangulations is always shape-regular in the following sense.

(A1) There exists $M_{1}<\infty$ such that any $T \in \mathcal{T} \in \mathbb{T}$ is included in a closed ball $\bar{B}\left(M_{T}, R_{T}\right)$ and includes a closed ball $\bar{B}\left(m_{T}, r_{T}\right)$ of radii $R_{T}$ and $r_{T}, \bar{B}\left(m_{T}, r_{T}\right) \subset T \subset \bar{B}\left(M_{T}, R_{T}\right)$, with $R_{T} \leq M_{1} r_{T}$. This implies finite overlap of patches and their extensions in that $|\mathcal{T}(z)| \leq M_{2}<$ $\infty$ for any $\mathcal{T} \in \mathbb{T}$ and $z \in \mathcal{N}$ and $M_{3}:=\sup _{T \in \mathcal{T} \in \mathbb{T}}|\mathcal{T}(\Omega(T))| \leq(n+1) M_{2}<\infty$. The constants $M_{1}, M_{2}, M_{3}$ are universal in $\mathbb{T}$.

Adaptive mesh-refinement typically leads to triangulations with a finite number of configurations up to scaling in the following sense. 
(A2) There exists a finite number of reference patches $\mathcal{C}_{1}, \ldots, \mathcal{C}_{J}$ of the vertex 0 such that for all $\mathcal{T} \in \mathbb{T}$ and any vertex $z \in \mathcal{N}$ the patch

$$
\mathcal{T}(z)=z+h \mathcal{C}_{j}
$$

is equal to a scaled copy of $\mathcal{C}_{j}$ for some $h>0$ and some $j \in\{1, \ldots, J\}$ and $h \mathcal{C}_{j}=\left\{h K: K \in \mathcal{C}_{j}\right\}$ with $h K=\{h x: x \in K\}$.

The most prominent mesh-refining strategy with (A2) is the newest vertex bisection (NVB) based on an initial triangulation $\mathcal{T}_{0}$ (plus some initialization of tagged simplices as in [25]). It is obvious that (A2) implies (A1).

Jumps. Given any side $F \in \mathcal{F}$, assign its unit normal $\nu_{F}$ with a fixed orientation, while $\nu_{T}$ denotes the unit outward normal along the simplex boundary $\partial T$ of $T \in \mathcal{T}$. Suppose $\nu_{F}=\left.\nu_{T}\right|_{F}$ on each boundary side $F \in \mathcal{F}(\partial \Omega) \cap \mathcal{F}(T)$. Once the orientation of the unit normal $\nu_{F}$ is fixed for an interior side $F=\partial T_{+} \cap \partial T_{-} \in \mathcal{F}(\Omega)$ shared by the simplices $T_{+}, T_{-} \in \mathcal{T}(F)$, let $T_{+}$ denote the adjoint simplex with $\left.\nu_{T_{+}}\right|_{F}=\nu_{F}$ and let $T_{-}$denote the simplex with $\left.\nu_{T_{-}}\right|_{F}=-\nu_{F}$. With this sign convention, the jump $[v]_{F}$ of a piecewise Lipschitz continuous function $v$ across $F$ is defined by

$$
[v]_{F}(x):= \begin{cases}\left.v\right|_{T_{+}}(x)-\left.v\right|_{T_{-}}(x) & \text { for } x \in F=\partial T_{+} \cap \partial T_{-} \in \mathcal{F}(\Omega), \\ v(x) & \text { for } x \in F \in \mathcal{F}(\partial \Omega) .\end{cases}
$$

General notation in $\mathbb{R}^{m \times k}$. For $a, b \in \mathbb{R}^{m \times k}$, let $a \cdot b=a^{\top} b \in \mathbb{R}^{k \times k}$ and $a \otimes b=a b^{\top} \in$ $\mathbb{R}^{m \times m}$. Let $e_{k} \in \mathbb{R}^{m}$ denote the canonical $k$-unit vector for $k=1, \ldots, m$ with $e_{k}(j)=\delta_{j k}$ for $1 \leq j, k \leq m$ and Kronecker delta $\delta_{j k}$. If $K=\operatorname{conv}\left\{P_{1}, P_{2}, \ldots, P_{J}\right\} \subset \mathbb{R}^{m}$, let $\operatorname{mid}(K):=$ $J^{-1} \sum_{j=1}^{J} P_{j} \in \mathbb{R}^{m}$ denote its centroid, e.g., the midpoint of an simplex, face or edge; set $h_{K}:=\operatorname{diam}(K)$.

The notation $|\cdot|$ depends on the context and denotes the euclidean length, the cardinality of a finite set, the $n$ - or $(n-1)$-dimensional Lebesgue measure of a subset of $\mathbb{R}^{n}$, e.g., $|T|$ is the volume of a simplex $T \in \mathcal{T}$ and $|F|$ denotes the area of a face $F \in \mathcal{F}$ in 3D or the length of an edge in $2 \mathrm{D}$.

Piecewise polynomials. The vector space of piecewise polynomials of at most degree $k$ is denoted by $P_{k}(\mathcal{T})$, the subset in $H^{1}(\Omega)$ by $S^{k}(\mathcal{T}):=P_{k}(\mathcal{T}) \cap C(\bar{\Omega}) \subset H^{1}(\Omega)$, and the subset in $H_{0}^{1}(\Omega)$ including homogeneous boundary conditions by $S_{0}^{k}(\mathcal{T}):=S^{k}(\mathcal{T}) \cap C_{0}(\Omega) \subset H_{0}^{1}(\Omega)$. Given a function $v \in L^{2}(\omega)$, define the integral mean $f_{\omega} v \mathrm{~d} x:=1 /|\omega| \int_{\omega} v \mathrm{~d} x$. The orthogonal projection $\Pi_{0}: L^{2}(\Omega) \rightarrow P_{0}(\mathcal{T})$ is defined for all $f \in L^{2}(\Omega)$ by its average $\left.\Pi_{0}(f)\right|_{T}:=f_{T} f \mathrm{~d} x$ in $T \in \mathcal{T}$.

\section{Abstract Discussion of Discrete Reliability}

\subsection{Goal}

It is the scope of this section to give an abstract and easy-to-read introduction to the principles of a proof of the discrete reliability (dRel) for nonconforming finite element methods. One key difficulty in the a posteriori error analysis of those methods results from the fact that even if the triangulation $\widehat{\mathcal{T}}$ is an admissible refinement of a regular triangulation $\mathcal{T}$, the related 
finite element spaces $V(\widehat{\mathcal{T}})$ and $V(\mathcal{T})$ are non-nested in that $V(\mathcal{T}) \not \subset V(\widehat{\mathcal{T}})$ in general. In comparison with nested conforming discretizations, this causes an additional a posteriori error term in (1.3) involving an approximation $\widehat{u}_{h}^{*} \in V(\widehat{\mathcal{T}})$ of the discrete solution $u_{h} \in V(\mathcal{T})$. The abstract description in this section introduces some general properties that cover the CrouzeixRaviart and the Morley finite element method. One key ingredient in the methodology (iii) for the definition of $\widehat{u}_{h}^{*}$ is the design of a conforming companion guided by $(\mathrm{C} 6)-(\mathrm{C} 7)$ and the consequence (3.4). In the abstract setting of this section, (1.4) and therefore (dRel) is proven for the set $\mathcal{R}$, which contains $\mathcal{T} \backslash \widehat{\mathcal{T}}$ plus one layer of simplices. A novel design of the companion operator in Section 6 allows the replacement of $\mathcal{R}$ by $\mathcal{T} \backslash \widehat{\mathcal{T}}$.

\subsection{Model problem}

To illustrate the proof of the discrete reliability (dRel), suppose that $\left(V(\mathcal{T}), a_{h}\right)$ is a finitedimensional Hilbert space based on a regular triangulation $\mathcal{T}$ of $\Omega \subset \mathbb{R}^{n}$, where $V(\mathcal{T}) \subset P_{k}(\mathcal{T})$ is a vector space of piecewise polynomials of degree at most $k$ and $a_{h}(\bullet, \bullet):=\left(D_{\mathrm{NC}}^{m} \cdot, D_{\mathrm{NC}}^{m} \bullet\right)$ is a scalar product that involves all piecewise derivatives $D_{\mathrm{NC}}^{m}$ of order $m$. In the case $m=1$, $D_{\mathrm{NC}}^{1}:=D_{\mathrm{NC}}=\nabla_{\mathrm{NC}}$ denotes the piecewise action of gradient $\nabla$, while $D_{\mathrm{NC}}^{2}$ stands for the piecewise action of the Hessian $D^{2}$ for $m=2$. The underlying triangulation is neither explicit in the notation of the scalar product $a_{h}$ nor in its induced norm $\|\cdot\|_{h}$ with

$$
\|\cdot\|_{h}^{2}:=\sum_{T \in \mathcal{T}}\left\|D_{\mathrm{NC}}^{m} \cdot\right\|_{L^{2}(T)}^{2},
$$

so both are defined for a nonconforming finite element space $V(\widehat{\mathcal{T}})$ with respect to any admissible refinement $\widehat{\mathcal{T}} \in \mathbb{T}(\mathcal{T})$ of $\mathcal{T}$. The conditions $(\mathrm{C} 1)-(\mathrm{C} 3)$ below imply a partial a posteriori error control exemplified in Theorem 3.1 for a linear model problem with $a_{h}$ and the right-hand side $f \in L^{2}(\Omega)$ with the associated functional $F(v):=\int_{\Omega} f v \mathrm{~d} x$ for $v \in L^{2}(\Omega)$. Let the discrete solution $u_{h} \in V(\mathcal{T})$ solve

$$
a_{h}\left(u_{h}, v_{h}\right)=F\left(v_{h}\right) \text { for all } v_{h} \in V(\mathcal{T}) .
$$

On the fine level, let $\widehat{u}_{h} \in V(\widehat{\mathcal{T}})$ denote the discrete solution to $a_{h}\left(\widehat{u}_{h}, \widehat{v}_{h}\right)=F\left(\widehat{v}_{h}\right)$ for all $\widehat{v}_{h} \in V(\widehat{\mathcal{T}})$. The local error estimator $\eta(T)$ from (1.5) leads for $\mathcal{M} \subseteq \mathcal{T}$ to

$$
\eta(\mathcal{T}, \mathcal{M}):=\sqrt{\sum_{K \in \mathcal{M}} \eta^{2}(K)}
$$

In (1.5), $\left[D^{m} u_{h}\right]_{F} \times \nu_{F}$ stands for the tangential components of the jump of the derivative $D^{m} u_{h}$ in $3 \mathrm{D}$ and simplifies to $\left[\partial u_{h} / \partial s\right]_{F}$ in $2 \mathrm{D}$ for $m=1$. The error estimator $\eta$ is reliable and efficient for a large class of examples [12].

\subsection{Conditions $(\mathrm{C} 1)-(\mathrm{C} 4)$}

Suppose that the nonconforming finite element space $V(\mathcal{T}) \not \subset H_{0}^{m}(\Omega)$ allows for an interpolation operator $I_{h}: H_{0}^{m}(\Omega)+V(\widehat{\mathcal{T}}) \rightarrow V(\mathcal{T})$ with an approximation property

$$
\left\|\widehat{v}_{h}-I_{h} \widehat{v}_{h}\right\|_{L^{2}(T)} \leq \Lambda_{1} h_{T}^{m}\left\|D^{m}\left(\widehat{v}_{h}-I_{h} \widehat{v}_{h}\right)\right\|_{L^{2}(T)} \quad \text { for all } T \in \mathcal{T}
$$

and an orthogonality

$$
a_{h}\left(w_{h}, \widehat{v}_{h}-I_{h} \widehat{v}_{h}\right)=0 \quad \text { for all } w_{h} \in V(\mathcal{T}) \text { and all } \widehat{v}_{h} \in V(\widehat{\mathcal{T}}) .
$$


Suppose the interpolation operator $I_{h}$ acts as the identity on non-refined simplices, in the sense that

$$
\left.\left(1-I_{h}\right) \widehat{v}_{h}\right|_{T}=0 \quad \text { in } T \in \mathcal{T} \cap \widehat{\mathcal{T}} \text { for all } \widehat{v}_{h} \in V(\widehat{\mathcal{T}}) .
$$

The point in what follows is that the non-nestedness $V(\mathcal{T}) \not \subset V(\widehat{\mathcal{T}})$ causes that $u_{h} \notin V(\widehat{\mathcal{T}})$ (in general) is not an admissible test function on the finer level. Some transfer function $\widehat{u}_{h}^{*} \in V(\widehat{\mathcal{T}})$ has to approximate $u_{h}$ in the norm of $L^{2}(\Omega)$ as well as in the norm $\|\cdot\|_{h}$ and results in estimator contributions for some simplices in $\mathcal{R} \subseteq \mathcal{T}$ below. The main argument for the later reduction to $\mathcal{R}$ is the property $\widehat{u}_{h}^{*}=u_{h}$ in $T \in \mathcal{T} \cap \widehat{\mathcal{T}}$. The introduction quotes a few references based on (i) appropriate mesh-refining and (ii) discrete Helmholtz decomposition to achieve this. Given $u_{h}$ on the coarse level, this paper suggests (iii) the design of $\widehat{u}_{h}^{*} \in V(\widehat{\mathcal{T}})$ on the fine level with

$$
I_{h} \widehat{u}_{h}^{*}=u_{h} .
$$

\subsection{Proof of (1.3)}

Theorem 3.1. The conditions $(\mathrm{C} 1)-(\mathrm{C} 4)$ imply (1.3) from the introduction.

Proof. The linearity of the discrete scalar product and (C2) imply

$$
\left\|\widehat{u}_{h}-u_{h}\right\|_{h}^{2}=a_{h}\left(\widehat{u}_{h}, \widehat{u}_{h}-u_{h}\right)-a_{h}\left(u_{h}, I_{h} \widehat{u}_{h}-u_{h}\right) .
$$

Given any $\widehat{u}_{h}^{*} \in V(\widehat{\mathcal{T}})$, the discrete equations on the coarse level with test-function $I_{h} \widehat{u}_{h}-u_{h} \in$ $V(\mathcal{T})$ and on the fine level with test-function $\widehat{u}_{h}-\widehat{u}_{h}^{*} \in V(\widehat{\mathcal{T}})$ lead to

$$
\left\|\widehat{u}_{h}-u_{h}\right\|_{h}^{2}=a_{h}\left(\widehat{u}_{h}, \widehat{u}_{h}^{*}-u_{h}\right)+F\left(\left(1-I_{h}\right)\left(\widehat{u}_{h}-\widehat{u}_{h}^{*}\right)+u_{h}-I_{h} \widehat{u}_{h}^{*}\right) .
$$

Since $\widehat{u}_{h}^{*} \in V(\widehat{\mathcal{T}})$ satisfies $(\mathrm{C} 4),(\mathrm{C} 2)$ implies $a_{h}\left(u_{h}, \widehat{u}_{h}^{*}-u_{h}\right)=0$. Therefore, the CauchySchwarz inequality and $(\mathrm{C} 1)-(\mathrm{C} 3)$ result in

$$
\begin{aligned}
\left\|\widehat{u}_{h}-u_{h}\right\|_{h}^{2} & =a_{h}\left(\widehat{u}_{h}-u_{h}, \widehat{u}_{h}^{*}-u_{h}\right)+F\left(\left(1-I_{h}\right)\left(\widehat{u}_{h}-\widehat{u}_{h}^{*}\right)\right) \\
& \leq\left\|\widehat{u}_{h}-u_{h}\right\|_{h}\left\|\widehat{u}_{h}^{*}-u_{h}\right\|_{h}+\Lambda_{1}\left\|h_{\mathcal{T}}^{m} f\right\|_{L^{2}(\mathcal{T} \backslash \widehat{\mathcal{T}})}\left\|\left(1-I_{h}\right)\left(\widehat{u}_{h}-\widehat{u}_{h}^{*}\right)\right\|_{h}
\end{aligned}
$$

with the abbreviation (for any $s \in \mathbb{R}$ )

$$
\left\|h_{\mathcal{T}}^{s} \cdot\right\|_{L^{2}(\mathcal{T} \backslash \widehat{\mathcal{T}})}:=\left(\sum_{T \in \mathcal{T} \backslash \widehat{\mathcal{T}}} h_{T}^{2 s}\|\cdot\|_{L^{2}(T)}^{2}\right)^{1 / 2} .
$$

The orthogonality $(\mathrm{C} 2)$ shows that

$$
\left\|\left(1-I_{h}\right)\left(\widehat{u}_{h}-\widehat{u}_{h}^{*}\right)\right\|_{h}^{2}=a_{h}\left(\left(1-I_{h}\right)\left(\widehat{u}_{h}-\widehat{u}_{h}^{*}\right), \widehat{u}_{h}-\widehat{u}_{h}^{*}\right) \leq\left\|\left(1-I_{h}\right)\left(\widehat{u}_{h}-\widehat{u}_{h}^{*}\right)\right\|_{h}\left\|\widehat{u}_{h}-\widehat{u}_{h}^{*}\right\|_{h} .
$$

This and the triangle inequality verify

$$
\left\|\left(1-I_{h}\right)\left(\widehat{u}_{h}-\widehat{u}_{h}^{*}\right)\right\|_{h} \leq\left\|\widehat{u}_{h}-u_{h}\right\|_{h}+\left\|\widehat{u}_{h}^{*}-u_{h}\right\|_{h} .
$$

The combination of (3.1)-(3.2) and some elementary calculations conclude the proof of (1.3). 


\subsection{Conditions $(\mathrm{C} 5)-(\mathrm{C} 7)$}

This section discusses the term $\left\|\widehat{u}_{h}^{*}-u_{h}\right\|_{h}$ and introduces additional conditions (C5)-(C7) sufficient for (1.4). The explicit design of $\widehat{u}_{h}^{*}$ in this paper (iii) involves a conforming companion $J_{2} u_{h} \in V_{C}(\mathcal{T}) \subset H_{0}^{m}(\Omega)$ followed by nonconforming interpolation $\widehat{I}_{h}: V_{C}(\mathcal{T}) \rightarrow V(\widehat{\mathcal{T}})$, namely

$$
\widehat{u}_{h}^{*}:=\widehat{I}_{h} J_{2} u_{h} .
$$

The conforming space $V_{C}(\mathcal{T})$ depends on the problem at hand; it is the conforming $V_{C}(\mathcal{T}):=$ $S_{0}^{n}(\mathcal{T})$ for $m=1$ and the Hsieh-Clough-Tocher finite element $V_{C}(\mathcal{T}):=H C T(\mathcal{T}) \subset H_{0}^{2}(\Omega)$ for $m=2=n$. More details for the two examples follow in Section 4 in (4.1)-(4.2) and in Section 5 in Lemma 5.1. Once $J_{2} u_{h} \in H_{0}^{m}(\Omega)$ is given, the stability of the nonconforming interpolation $\widehat{I_{h}}: H_{0}^{m}(\Omega) \rightarrow V(\widehat{\mathcal{T}})$ leads to an universal constant $\Lambda_{5} \lesssim 1$ such that, for all $T \in \mathcal{T}$,

$$
\left\|D_{\mathrm{NC}}^{m}\left(\widehat{I_{h}} v-w_{h}\right)\right\|_{L^{2}(T)} \leq \Lambda_{5}\left\|D^{m}\left(v-w_{h}\right)\right\|_{L^{2}(T)} \text { for all } v \in H_{0}^{m}(\Omega) \text { and } w_{h} \in V(\mathcal{T}) .
$$

The combination of (C3)-(C5) with $v=J_{2} u_{h}, \widehat{u}_{h}^{*}=\widehat{I}_{h} J_{2} u_{h}$, and $\|\cdot\|_{h}=\left\|D_{\mathrm{NC}}^{m} \bullet\right\|_{L^{2}(\Omega)}$ proves

$$
\left\|\widehat{u}_{h}^{*}-u_{h}\right\|_{h} \leq \Lambda_{5}\left\|D^{m}\left(J_{2} u_{h}-u_{h}\right)\right\|_{L^{2}(\mathcal{T} \backslash \widehat{\mathcal{T}})} .
$$

The subsequent discussion concerns the local analysis of the upper bound $\left\|D^{m}\left(J_{2} u_{h}-u_{h}\right)\right\|_{L^{2}(T)}$ for $T \in \mathcal{T} \backslash \widehat{\mathcal{T}}$ and that means the design of $J_{2}$. The abstract description of the local design of $J_{2}: V(\mathcal{T}) \rightarrow V_{C}(\mathcal{T})$ in $(\mathrm{C} 6)$ below assumes that $\left.\left(J_{2} v_{h}\right)\right|_{T}$ depends on $v_{h} \in V(\mathcal{T})$ restricted to a neighbourhood $\Omega(T)$ of $T \in \mathcal{T}$. In a formal notation, for all $T \in \mathcal{T}$ with $\left.V(\mathcal{T})\right|_{\Omega(T)}:=\left\{\left.v_{h}\right|_{\Omega(T)}\right.$ : $\left.v_{h} \in V(\mathcal{T})\right\} \subset P_{k}(\mathcal{T}(\Omega(T)))$ and $\left.\left(V(\mathcal{T}) \cap H_{0}^{m}(\Omega)\right)\right|_{\Omega(T)}:=\left\{\left.v\right|_{\Omega(T)}: v \in V(\mathcal{T}) \cap H_{0}^{m}(\Omega)\right\}$, assume the existence of an operator $J_{2, T}:\left.V(\mathcal{T})\right|_{\Omega(T)} \rightarrow V_{C}(\mathcal{T})$ with

$$
\left.\left(J_{2} v_{h}\right)\right|_{T}=J_{2, T}\left(\left.v_{h}\right|_{\Omega(T)}\right) \quad \text { for all } v_{h} \in V(\mathcal{T}) .
$$

This local contribution $J_{2, T}$ is exact for all conforming arguments in the sense that

$$
\left.w_{h}\right|_{T}=J_{2, T}\left(w_{h}\right) \quad \text { for all }\left.w_{h} \in\left(V(\mathcal{T}) \cap H_{0}^{m}(\Omega)\right)\right|_{\Omega(T)} \text { and all } T \in \mathcal{T} .
$$

The jump estimator contributions near some simplex $T \in \mathcal{T}$ are associated with the set $\mathcal{F}(\Omega(T))$ of sides, which is defined as the set of all $F=\partial K_{1} \cap \partial K_{2}$ for distinct neighbouring simplices $K_{1}, K_{2} \in \mathcal{T}(\Omega(T))$ plus all boundary sides $F \subseteq \partial \Omega$ with $F \in \mathcal{F}(K)$ for some $K \in \mathcal{T}(\Omega(T))$. (Notice that any side $F$ on the boundary $\partial(\Omega(T))$ of $\Omega(T)$ is only included if it belongs to $\partial \Omega$; if $\operatorname{dist}(\Omega(T), \partial \Omega)>0$ then only interior sides in $\Omega(T)$ are considered in $\mathcal{F}(\Omega(T))$.) Define the two seminorms $\mu_{T}, \varrho_{T}:\left.V(\mathcal{T})\right|_{\Omega(T)} \rightarrow[0, \infty)$ for $\left.w_{h} \in V(\mathcal{T})\right|_{\Omega(T)}=\left\{\left.v_{h}\right|_{\Omega(T)}: v_{h} \in V(\mathcal{T})\right\}$ by

$$
\begin{aligned}
\mu_{T}\left(w_{h}\right) & :=\left(\sum_{F \in \mathcal{F}(\Omega(T))} h_{F}\left\|\left[D^{m} w_{h}\right]_{F} \times \nu_{F}\right\|_{L^{2}(F)}^{2}\right)^{1 / 2} \text { and } \\
\varrho_{T}\left(w_{h}\right) & :=\left\|D^{m}\left(w_{h}-J_{2, T} w_{h}\right)\right\|_{L^{2}(T)} .
\end{aligned}
$$

The condition $(\mathrm{C} 6)$ implies that $\left.\left(V(\mathcal{T}) \cap H_{0}^{m}(\Omega)\right)\right|_{\Omega(T)}$ belongs to the null space

$$
\operatorname{Ker} \varrho_{T}=\left\{\left.w_{h} \in V(\mathcal{T})\right|_{\Omega(T)}: \varrho_{T}\left(w_{h}\right)=0\right\}
$$

of $\varrho_{T}$. The latter space is supposed to include the null space $\operatorname{Ker} \mu_{T}$ of $\mu_{T}$ in that

$$
\left.\forall w_{h} \in V(\mathcal{T})\right|_{\Omega(T)} \quad\left(\mu_{T}\left(w_{h}\right)=\left.0 \quad \Rightarrow \quad w_{h} \in\left(V(\mathcal{T}) \cap H_{0}^{m}(\Omega)\right)\right|_{\Omega(T)}\right) .
$$


In conclusion, $\operatorname{Ker} \mu_{T} \subset \operatorname{Ker} \varrho_{T}$. The vector space $\left.V(\mathcal{T})\right|_{\Omega(T)}$ has dimension at most $\operatorname{dim} P_{k}(T)=$ $\left(\begin{array}{c}k+n \\ n\end{array}\right)$ times the cardinality $|\mathcal{T}(\Omega(T))| \leq M_{3}$ of simplices near $T$. Hence, an inverse estimate argument similar to that in the proof of the equivalence of norms on a finite-dimensional vector space $\left.V(\mathcal{T})\right|_{\Omega(T)}$ leads to

$$
\varrho_{T}\left(w_{h}\right) \leq C(T) \mu_{T}\left(w_{h}\right) \quad \text { for all }\left.w_{h} \in V(\mathcal{T})\right|_{\Omega(T)}
$$

for some constant $C(T)$ that depends on the local companion operator $J_{2, T}$, the triangulation $\mathcal{T}(\Omega(T)$ ), the sides $\mathcal{F}(\Omega(T)$ ), and the maximal polynomial degree $k$. Under the assumption (A2) on $\mathbb{T}$, the constants $C(T)$ in (3.4) are uniformly bounded.

Lemma 3.1. The assumptions (A2) and $(\mathrm{C} 6)-(\mathrm{C} 7)$ imply

$$
C(\mathbb{T}):=\sup _{T \in \mathcal{T} \in \mathbb{T}} C(T)<\infty .
$$

Proof. The aforementioned soft analysis arguments lead to (3.4) with a constant $C(T)$ that depends on the maximal polynomial degree $k$ and on the configuration $\mathcal{T}(\Omega(T)$ ). The assumption (A2) states that any nodal patch $\mathcal{T}(z)$ is equal to $z+h_{z} \mathcal{C}_{j(z)}$ for some $j(z) \in\{1, \ldots, J\}$ and some $h_{z}>0$. Since $\mathcal{T}(\Omega(T))$ is the union of the $n+1$ nodal patches for the vertices $z \in \mathcal{N}(T)$ of $T$, it follows

$$
\mathcal{T}(\Omega(T))=\bigcup_{z \in \mathcal{N}(T)}\left(z+h_{z} \mathcal{C}_{j(z)}\right)
$$

A scaling argument of the piecewise polynomials shows that the constant $C(T)$ does not depend on a uniform scaling of all those factors $\left\{h_{z}: z \in \mathcal{N}(T)\right\}$, so without loss of generality let $h_{T}=1$. Then the other scaling factors are determined by the shape-regularity of $\mathcal{T}(\Omega(T))$ and their overlap $T$; in other words, there exists only a finite number of (scaled) configurations $\mathcal{T}\left(\Omega(T)\right.$ ) with $h_{T}=1$ despite the fact that there are infinite triangulations $\mathcal{T}$ in $\mathbb{T}$. Each of those configurations leads to some positive constant $C(T)$ and the maximum of those finite number of values is $C(\mathbb{T})$, which is positive and exclusively depends on $\mathbb{T}$ and on the maximal polynomial degree $k$. This concludes the proof.

Lemma 3.1 shows that the general assumptions $(\mathrm{C} 6)-(\mathrm{C} 7)$ and (A2) are one example for sufficient conditions for (3.4)-(3.5). For nonconforming Crouzeix-Raviart and Morley finite element methods, the subsequent sections present some upper bounds of $C(\mathbb{T})$ for $n=2$ and show that $C(\mathbb{T})>0$ depends solely on the minimal angle $\omega_{0}$ in $\mathbb{T}$ from (A1).

\subsection{Proof of (1.4)}

Theorem 3.2. The assumptions (A2) and (C3)-(C7) imply (1.4) with $\Lambda_{2}=\Lambda_{5} C(\mathbb{T}) M_{3}^{1 / 2}$.

Proof. Recall that a combination of (C3)-(C4) shows

$$
\left.\widehat{u}_{h}^{*}\right|_{T}=\left.I_{h} \widehat{u}_{h}^{*}\right|_{T}=\left.u_{h}\right|_{T} \text { for } T \in \mathcal{T} \cap \widehat{\mathcal{T}}
$$

and then (C5) implies (3.3). The definitions of $\mu_{T}$ and $\varrho_{T}$ lead in Lemma 3.1 to (3.4)-(3.5),

$$
\left\|D^{m}\left(w_{h}-J_{2, T} w_{h}\right)\right\|_{L^{2}(T)} \leq C(\mathbb{T}) \mu_{T}\left(w_{h}\right) \quad \text { for all } T \in \mathcal{T} \text { and all }\left.w_{h} \in V(\mathcal{T})\right|_{\Omega(T)} .
$$


Given any $v_{h} \in V(\mathcal{T})$, the piecewise definition of $J_{2}$ through the local contributions $J_{2, T}$ for $T \in \mathcal{T}$ and (3.7) for $w_{h}=\left.v_{h}\right|_{\Omega(T)}$ result in

$$
\begin{aligned}
& \sum_{T \in \mathcal{T} \backslash \widehat{\mathcal{T}}}\left\|D^{m}\left(v_{h}-J_{2} v_{h}\right)\right\|_{L^{2}(T)}^{2} \leq C(\mathbb{T})^{2} \sum_{T \in \mathcal{T} \backslash \widehat{\mathcal{T}}} \mu_{T}^{2}\left(\left.v_{h}\right|_{\Omega(T)}\right) \\
\leq & C(\mathbb{T})^{2} M_{3} \sum_{T \in \mathcal{R}} \sum_{F \in \mathcal{F}(T)} h_{F}\left\|\left[D^{m} v_{h}\right]_{F} \times \nu_{F}\right\|_{L^{2}(F)}^{2} .
\end{aligned}
$$

Since $F \in \mathcal{F}(T)$ implies $h_{F} \leq h_{T}$, this concludes the proof of (1.4).

\section{Crouzeix-Raviart Finite Elements}

This section establishes the conditions $(\mathrm{C} 1)-(\mathrm{C} 7)$ for Crouzeix-Raviart finite elements for $m=1$ and $n \geq 2$, hence with a second-order problem as the Poisson Model Problem (1.1) in mind. The notation from the abstract Section 3 is specified for the Crouzeix-Raviart finite element method in that $u_{C R}$ replaces $u_{h}, I_{\mathrm{NC}}$ replaces $I_{h}$, and $\sum_{T \in \mathcal{T}}\left\|\nabla_{\mathrm{NC}} \bullet\right\|_{L^{2}(T)}^{2}$ replaces $\|\cdot\|_{h}^{2}$ etc.

\subsection{Interpolation and Conforming Companion Operator}

The Crouzeix-Raviart finite element spaces (with and without boundary conditions) read

$$
\begin{aligned}
& C R^{1}(\mathcal{T}):=\left\{v_{C R} \in P_{1}(\mathcal{T}): v_{C R} \text { is continuous at } \operatorname{mid}(F) \text { for all } F \in \mathcal{F}(\Omega)\right\}, \\
& C R_{0}^{1}(\mathcal{T}):=\left\{v_{C R} \in C R^{1}(\mathcal{T}): v_{C R}(\operatorname{mid}(F))=0 \text { for all } F \in \mathcal{F}(\partial \Omega)\right\} .
\end{aligned}
$$

For any admissible refinement $\hat{\mathcal{T}} \in \mathbb{T}(\mathcal{T})$ of $\mathcal{T} \in \mathbb{T}$ and the side-oriented basis functions $\psi_{F} \in$ $C R^{1}(\mathcal{T})$ with $\psi_{F}(\operatorname{mid}(E))=\delta_{E F}$ for all sides $E, F \in \mathcal{F}$, the interpolation operator $I_{\mathrm{NC}}$ : $H_{0}^{1}(\Omega)+C R_{0}^{1}(\widehat{\mathcal{T}}) \rightarrow C R_{0}^{1}(\mathcal{T})$ reads

$$
I_{\mathrm{NC}}(f):=\sum_{F \in \mathcal{F}}\left(f_{F} f \mathrm{~d} s\right) \psi_{F} \quad \text { for any } f \in H_{0}^{1}(\Omega)+C R_{0}^{1}(\widehat{\mathcal{T}}) .
$$

The side-oriented basis functions $\left(\widehat{\psi}_{F}: F \in \widehat{\mathcal{F}}\right)$ of $C R^{1}(\widehat{\mathcal{T}})$ with respect to the fine triangulation $\widehat{\mathcal{T}}$ allow the analog definition of the interpolation operator $\widehat{I}_{\mathrm{NC}}: H_{0}^{1}(\Omega) \rightarrow C R_{0}^{1}(\widehat{\mathcal{T}})$.

The design for $\Omega \subset \mathbb{R}^{2}$ of the conforming companion operator $J_{2}: C R_{0}^{1}(\mathcal{T}) \rightarrow S_{0}^{2}(\mathcal{T}) \subset$ $H_{0}^{1}(\Omega)$ from [8, Proof of Prop.2.3] generalizes to any space dimension $n \geq 2$. Let $\left.v_{C R}\right|_{T}(z)$ denote the value of $v_{C R} \in C R_{0}^{1}(\mathcal{T})$ on $T \in \mathcal{T}$ at the vertex $z \in \mathcal{N}(T)$ and let $|\mathcal{T}(z)| \geq 1$ be the number of simplices in the nodal patch. Nodal averaging defines $J_{1}: C R_{0}^{1}(\mathcal{T}) \rightarrow S_{0}^{1}(\mathcal{T})$, where

$$
\left(J_{1} v_{C R}\right)(z)=\left.|\mathcal{T}(z)|^{-1} \sum_{T \in \mathcal{T}(z)} v_{C R}\right|_{T}(z) \quad \text { for all } z \in \mathcal{N}(\Omega)
$$

is followed by linear interpolation (plus homogeneous boundary conditions). This is called an enrichment operator in [3] and also considered in the medius analysis in [14,19]. Let $\varphi_{z} \in S^{1}(\mathcal{T})$ with $\varphi_{z}(a)=\delta_{a z}$ for all vertices $a, z \in \mathcal{N}$ denote the $P_{1}$-conforming basis functions and let $b_{F}:=\left(\prod_{z \in \mathcal{N}(F)} \varphi_{z}\right) / \int_{F}\left(\prod_{z \in \mathcal{N}(F)} \varphi_{z}\right) \mathrm{d} s \in P_{n}(\mathcal{T}(F))$ for any side $F \in \mathcal{F}$ be a normalized side-bubble function. Then $J_{2}: C R_{0}^{1}(\mathcal{T}) \rightarrow S_{0}^{n}(\mathcal{T})$ reads

$$
J_{2} v_{C R}:=J_{1} v_{C R}+\sum_{F \in \mathcal{F}(\Omega)}\left(f_{F}\left(v_{C R}-J_{1} v_{C R}\right) \mathrm{d} s\right) b_{F} .
$$




\subsection{Proof of $(\mathrm{C} 1)$}

This is Theorem 3.5 in [10] with $\Lambda_{1}=\sqrt{19 / 48} \leq 0.629153$ for $n=2$ or $\Lambda_{1}=\sqrt{101 / 180} \leq$ 0.749074 for $n=3$.

\subsection{Proof of $(\mathrm{C} 2),(\mathrm{C} 5)$}

Lemma 13 in [11] recalls $\Pi_{0} \nabla_{\mathrm{NC}} \widehat{v}_{C R}=\nabla_{\mathrm{NC}} I_{\mathrm{NC}} \widehat{v}_{C R} \in P_{0}\left(\mathcal{T} ; \mathbb{R}^{n}\right)$ for all $\widehat{v}_{C R} \in C R_{0}^{1}(\widehat{\mathcal{T}})$. Since $\nabla_{\mathrm{NC}} w_{C R} \in P_{0}\left(\mathcal{T} ; \mathbb{R}^{n}\right)$ for all $w_{C R} \in C R_{0}^{1}(\mathcal{T}),(\mathrm{C} 2)$ follows from

$$
a_{h}\left(w_{C R}, \widehat{v}_{C R}-I_{\mathrm{NC}} \widehat{v}_{C R}\right)=\left(\nabla_{\mathrm{NC}} w_{C R},\left(1-\Pi_{0}\right) \nabla_{\mathrm{NC}} \widehat{v}_{C R}\right)_{L^{2}(\Omega)}=0 .
$$

The analog identity on the refined triangulation $\widehat{\mathcal{T}}$ reads $\widehat{\Pi}_{0} \nabla v=\nabla_{\mathrm{NC}} \widehat{I}_{\mathrm{NC}} v$ for all $v \in H_{0}^{1}(\Omega)$. This and $\nabla_{\mathrm{NC}} w_{C R} \in P_{0}\left(\mathcal{T} ; \mathbb{R}^{n}\right) \subset P_{0}\left(\widehat{\mathcal{T}} ; \mathbb{R}^{n}\right)$ imply $(\mathrm{C} 5)$ for any $T \in \mathcal{T}$ with $\Lambda_{5}=1$.

\subsection{Proof of $(\mathrm{C} 3)$}

The restriction of any $\widehat{v}_{C R} \in C R_{0}^{1}(\widehat{\mathcal{T}})$ to some $T \in \mathcal{T} \cap \widehat{\mathcal{T}}$ satisfies $\left.\widehat{v}_{C R}\right|_{T} \in \operatorname{span}\left\{\left.\psi_{F}\right|_{T}: F \in\right.$ $\mathcal{F}(T)\}$ with the side-oriented shape functions $\psi_{F} \in C R_{0}^{1}(\mathcal{T})$. The duality property $f_{F} \psi_{E} \mathrm{~d} s=$ $\delta_{E F}$ for all sides $E, F \in \mathcal{F}$ implies $\left.I_{\mathrm{NC}} \widehat{v}_{C R}\right|_{T}=\left.\widehat{v}_{C R}\right|_{T}$.

\subsection{Proof of $(\mathrm{C} 4)$}

Given any $u_{C R} \in C R_{0}^{1}(\mathcal{T})$, set $\widehat{u}_{C R}^{*}:=\widehat{I}_{\mathrm{NC}} J_{2}\left(u_{C R}\right)$. The correction with normalized sidebubble functions in (4.2) guarantees $f_{F} J_{2} u_{C R} \mathrm{~d} s=f_{F} u_{C R} \mathrm{~d} s$ for all sides $F \in \mathcal{F}$. Hence, the definition of $I_{\mathrm{NC}}$ implies $I_{\mathrm{NC}} \widehat{u}_{C R}^{*}=I_{\mathrm{NC}} J_{2}\left(u_{C R}\right)=I_{\mathrm{NC}} u_{C R}=u_{C R}$. This proves $(\mathrm{C} 4)$.

\subsection{Proof of $(\mathrm{C} 6)$}

Given any $v_{C R} \in C R_{0}^{1}(\mathcal{T})$ and $K \in \mathcal{T}$, the restriction $\left.J_{1} v_{C R}\right|_{K}$ of the conforming companion $J_{1} v_{C R}$ is the linear interpolation of the nodal values $\left(J_{1} v_{C R}\right)(z)$ at $z \in \mathcal{N}(K) \cap \mathcal{N}(\Omega)$ computed from the nodal values of $\left.v_{C R}\right|_{T}(z)$ restricted to the simplex $T \in \mathcal{T}(z) \subset \mathcal{T}(\Omega(K))$. Therefore $J_{2}$ is associated with local contributions $J_{2, K}$ for any $K \in \mathcal{T}$ in the sense that

$$
\left.\left(J_{2} v_{C R}\right)\right|_{K}=J_{2, K}\left(\left.v_{C R}\right|_{\Omega(K)}\right) \quad \text { for all } v_{C R} \in C R_{0}^{1}(\mathcal{T}) .
$$

Any $\left.w_{C R} \in\left(C R_{0}^{1}(\mathcal{T}) \cap H_{0}^{1}(\Omega)\right)\right|_{\Omega(K)}=\left.S_{0}^{1}(\mathcal{T})\right|_{\Omega(K)}$ is continuous in $\Omega(K)$ and vanishes along $\partial \Omega \cap \partial(\Omega(K))$ so the values $J_{1}\left(w_{C R}\right)(z)=w_{C R}(z)$ coincide at all vertices $z \in \mathcal{N}(\Omega(K)):=$ $\{z \in \mathcal{N}(T): T \in \mathcal{T}(\Omega(K))\}$ and the integral means $f_{F}\left(w_{C R}-J_{1} w_{C R}\right) \mathrm{d} s=0$ vanish along all sides $F \in \mathcal{F}(\Omega(K))$. Consequently, for all $K \in \mathcal{T}, J_{2, K}$ satisfies (C6).

\subsection{Proof of $(\mathrm{C} 7)$}

Any $\left.w_{C R} \in C R_{0}^{1}(\mathcal{T})\right|_{\Omega(T)}$ is piecewise affine, continuous at the side midpoints and vanishes at midpoints of boundary sides $F \subseteq \partial \Omega \cap \partial(\Omega(T))$. Hence, the jump $\left[w_{C R}\right]_{F}$ across each side $F \in \mathcal{F}$ is of the form $\left[w_{C R}\right]_{F}(x)=a \cdot(x-\operatorname{mid}(F))$ for some $a \in \mathbb{R}^{n}$ and any $x \in F$. Since $a=\left[\nabla_{\mathrm{NC}} w_{C R}\right]_{F}$ and the normal $\nu_{F}$ is perpendicular to $(x-\operatorname{mid}(F)) \perp \nu_{F}$ at any $x \in F$, the jumps $\left[w_{C R}\right]_{F} \equiv 0$ vanish if and only if the tangential jumps of the gradients $\left\|\left[\nabla w_{C R}\right]_{F} \times \nu_{F}\right\|_{L^{2}(F)}^{2}=0$ vanish. Therefore, $\sum_{F \in \mathcal{F}(\Omega(T))} h_{F}\left\|\left[\nabla w_{C R}\right]_{F} \times \nu_{F}\right\|_{L^{2}(F)}^{2}=0$ implies that $w_{C R}$ is continuous in $\Omega(T)$ and vanishes along each boundary side $F \in \mathcal{F}(\partial \Omega) \cap \mathcal{F}(\partial \Omega(T))$. This proves $(\mathrm{C} 7)$. 


\subsection{Constants in $2 \mathrm{D}$}

In the case $\Omega \subset \mathbb{R}^{2}$, Section 4.2 shows $\Lambda_{1}=\sqrt{19 / 48} \leq 0.629153$ and this section bounds the constant $\Lambda_{2}$ in terms of the smallest angle $\omega_{0}$ in the set of admissible triangulations $\mathbb{T}$ and $M_{2}=\max _{\mathcal{T} \in \mathbb{T}}\{|\mathcal{T}(z)|: z \in \mathcal{N}\} \leq 2 \pi / \omega_{0}$. The combination of (3.3) and the inverse estimate [3, Lemma 4.5.3] with constant $c_{\text {inv }, 2}$ for piecewise polynomials of degree at most 2 implies

$$
\left\|\widehat{u}_{C R}^{*}-u_{C R}\right\|_{h} \leq c_{\mathrm{inv}, 2}\left\|h_{\mathcal{T}}^{-1}\left(u_{C R}-J_{2} u_{C R}\right)\right\|_{L^{2}(\mathcal{T} \backslash \widehat{\mathcal{T}})}
$$

For each $T \in \mathcal{T}$, the definition of $J_{2}$ in (4.2) and the triangle inequality lead to

$$
\begin{gathered}
\left\|u_{C R}-J_{2} u_{C R}\right\|_{L^{2}(T)} \\
\leq\left\|u_{C R}-J_{1} u_{C R}\right\|_{L^{2}(T)}+\left\|\sum_{F \in \mathcal{F}(T) \cap \mathcal{F}(\Omega)}\left|f_{F}\left(u_{C R}-J_{1} u_{C R}\right) \mathrm{d} s\right| b_{F}\right\|_{L^{2}(T)} .
\end{gathered}
$$

Moreover, the local mass matrix for normalized bubble functions reads (with unit matrix $1_{3 \times 3} \in$ $\mathbb{R}^{3 \times 3}$ )

$$
B(T):=\left(\int_{T} b_{E} b_{F} \mathrm{~d} x\right)_{E, F \in \mathcal{F}(T)}=\frac{|T|}{5}\left(1_{3 \times 3}+(1,1,1) \otimes(1,1,1)\right) \in \mathbb{R}^{3 \times 3}
$$

and has the double eigenvalue $\lambda_{\min }=|T| / 5$ and the simple eigenvalue $\lambda_{\max }=4|T| / 5$. The discrete trace identity $\sum_{F \in \mathcal{F}(T)}\left|f_{F} u_{C R}-J_{1} u_{C R} \mathrm{~d} s\right|^{2}=3|T|^{-1}\left\|u_{C R}-J_{1} u_{C R}\right\|_{L^{2}(T)}^{2}$ holds in 2D. Consequently,

$$
\left\|u_{C R}-J_{2} u_{C R}\right\|_{L^{2}(T)} \leq C_{J}\left\|u_{C R}-J_{1} u_{C R}\right\|_{L^{2}(T)} \text { with } C_{J}=1+2 \sqrt{3 / 5} \leq 2.5492
$$

Theorem 4.5 and Remark 4.7 in [10] prove that $C_{\text {loc }}:=\left(16 \sqrt{3}\left(1-\cos \left(\pi / M_{2}\right)\right)\right)^{-1}$ and $\eta_{F}^{2}:=$ $h_{F}\left\|\left[\nabla u_{C R}\right]_{F} \times \nu_{F}\right\|_{L^{2}(F)}^{2}$ satisfy

$$
h_{T}^{-2}\left\|u_{C R}-J_{1} u_{C R}\right\|_{L^{2}(T)}^{2} \leq C_{\mathrm{loc}} \sum_{z \in \mathcal{N}(T)} \sum_{\substack{F \in \mathcal{F} \\ z \in \mathcal{N}(F)}} \eta_{F}^{2} \leq 2 C_{\mathrm{loc}} \sum_{F \in \mathcal{F}(\Omega(T))} \eta_{F}^{2} .
$$

The combination of (4.3)-(4.5) proves $C(\mathbb{T}) \leq c_{\mathrm{inv}, 2} C_{J} \sqrt{2 C_{\text {loc }}}$ in (3.5). Furthermore, $M_{3} \leq$ $3 M_{2} \leq 6 \pi / \omega_{0}$, and $\Lambda_{2}=C(\mathbb{T}) M_{3}^{1 / 2}$ hold in (1.4).

\section{Morley Finite Elements}

This section verifies the conditions $(\mathrm{C} 1)-(\mathrm{C} 7)$ for Morley finite elements with $m=2=n$, hence with a fourth-order problem as the Biharmonic problem (1.2) in mind. The notation from the abstract Section 3 is adapted to the Morley finite element space in that $u_{M}$ replaces $u_{h}, I_{M}$ replaces $I_{h}$, and $\sum_{T \in \mathcal{T}}\left\|D_{\mathrm{NC}}^{2} \cdot\right\|_{L^{2}(T)}^{2}$ replaces $\|\cdot\|_{h}^{2}$ etc. 


\subsection{Interpolation and Conforming Companion Operator}

Given a regular triangulation $\mathcal{T}$ of $\Omega \subset \mathbb{R}^{2}$ with the set $\mathcal{E}$ of edges, the triangular equilibrium [22] also known as Morley finite element spaces (with and without boundary condition) is

$$
\begin{aligned}
& M^{\prime}(\mathcal{T}):=\left\{v \in P_{2}(\mathcal{T}): v \text { is continuous at } \mathcal{N}\right. \text { and } \\
& \left.\nabla_{\mathrm{NC}} v \text { is continuous at } \operatorname{mid}(E) \text { for all } E \in \mathcal{E}\right\}, \\
& M(\mathcal{T}):=\left\{v \in M^{\prime}(\mathcal{T}): v \text { vanisches at } \mathcal{N}(\partial \Omega)\right. \text { and } \\
& \left.\nabla_{\mathrm{NC}} v \text { vanishes at } \operatorname{mid}(E) \text { for all } E \in \mathcal{E}(\partial \Omega)\right\} \text {. }
\end{aligned}
$$

The shape functions for this finite element are displayed in $[6,(6.1)]$, the local degrees of freedom for $\phi_{M} \in M(\mathcal{T})$ on $T \in \mathcal{T}$ are the nodal values $\phi_{M}(z)$ for $z \in \mathcal{N}(T)$ and the normal derivatives $\partial \phi_{M} / \partial \nu_{E}(\operatorname{mid}(E))$ in the midpoints of the edges $E \in \mathcal{E}(T)$. For any admissible refinement $\widehat{\mathcal{T}} \in \mathbb{T}(\mathcal{T})$ of $\mathcal{T} \in \mathbb{T}$ and the normal derivative $\partial v / \partial \nu_{E}:=\nabla v \cdot \nu_{E}$ along the edges $E \in \mathcal{E}$, the interpolation operator $I_{M}: H_{0}^{2}(\Omega)+M(\widehat{\mathcal{T}}) \rightarrow M(\mathcal{T})[5,18]$ for any $v \in H_{0}^{2}(\Omega)+M(\widehat{\mathcal{T}})$ is characterized by

$$
\left(I_{M} v\right)(z)=v(z) \quad \text { for any } z \in \mathcal{N} \quad \text { and } \quad \frac{\partial I_{M} v}{\partial \nu_{E}}(\operatorname{mid}(E))=f_{E} \frac{\partial v}{\partial \nu_{E}} \mathrm{~d} s \quad \text { for any } E \in \mathcal{E} .
$$

The analog characterization with respect to the fine triangulation $\widehat{\mathcal{T}}$ defines the interpolation operator $\widehat{I}_{M}: H_{0}^{2}(\Omega) \rightarrow M(\widehat{\mathcal{T}})$ to the Morley finite element space $M(\widehat{\mathcal{T}})$.

A conforming finite-dimensional subspace of $H_{0}^{2}(\Omega)$ is the Hsieh-Clough-Tocher $(H C T)$ finite element [16, Chap. 6]. For any $T \in \mathcal{T}$ let $\mathcal{K}(T):=\left\{T_{E}: E \in \mathcal{E}(T)\right\}$ denote the triangulation of $T$ into three sub-triangles $T_{E}:=\operatorname{conv}\{E, \operatorname{mid}(T)\}$ with edges $E \in \mathcal{E}(T)$ and common vertex $\operatorname{mid}(T)$. Then,

$$
H C T(\mathcal{T}):=\left\{v \in H_{0}^{2}(\Omega):\left.v\right|_{T} \in P_{3}(\mathcal{K}(T)) \text { for all } T \in \mathcal{T}\right\} .
$$

The local degrees of freedom for $\psi \in H C T(\mathcal{T})$ on $T \in \mathcal{T}$ are the nodal values of the function $\psi(z)$, of the derivative $\nabla \psi(z)$ for $z \in \mathcal{N}(T)$ and the values of the normal derivatives $\partial \psi / \partial \nu_{E}(\operatorname{mid}(E))$ at the midpoints of the edges $E \in \mathcal{E}(T)$.

Lemma 5.1. There exists a conforming companion operator $J_{G}: M(\mathcal{T}) \rightarrow H C T(\mathcal{T})+\left(P_{5}(\mathcal{T}) \cap\right.$ $\left.H_{0}^{2}(\Omega)\right)$ such that $J_{G} v_{M} \in H C T(\mathcal{T})+\left(P_{5}(\mathcal{T}) \cap H_{0}^{2}(\Omega)\right)$ satisfies $(\mathrm{i})-(\mathrm{v})$ for any $v_{M} \in \mathcal{M}(\mathcal{T})$.

(i) $J_{G} v_{M}(z)=v_{M}(z)$ for any $z \in \mathcal{N}$;

(ii) $\nabla\left(J_{G} v_{M}\right)(z)= \begin{cases}|\mathcal{T}(z)|^{-1} \sum_{T \in \mathcal{T}(z)}\left(\left.\nabla v_{M}\right|_{T}\right)(z) & \text { for } z \in \mathcal{N}(\Omega), \\ 0 & \text { for } z \in \mathcal{N}(\partial \Omega) ;\end{cases}$

(iii) $f_{E} \partial J_{G} v_{M} / \partial \nu_{E} \mathrm{~d} s=f_{E} \partial v_{M} / \partial \nu_{E} \mathrm{~d} s$ for any $E \in \mathcal{E}$;

(iv) $I_{M} J_{G} v_{M}=v_{M}$;

(v) $h_{\mathcal{T}}^{-4}\left\|v_{M}-J_{G} v_{M}\right\|_{L^{2}(T)}^{2}$

$$
\lesssim \sum_{E \in \mathcal{E}(\Omega(T))} h_{E}\left\|\left[D^{2} v_{M}\right]_{E} \times \nu_{E}\right\|_{L^{2}(E)}^{2} \lesssim \min _{v \in H_{0}^{2}(\Omega)}\left\|D_{\mathrm{NC}}^{2}\left(v_{M}-v\right)\right\|_{L^{2}(\Omega(T))}^{2}
$$


Proof. Proposition 2.5 of [18] defines a companion operator with (i)-(ii). In that paper given $v_{M} \in M(\mathcal{T})$, the first step is the definition of some $J_{1} v_{M} \in H C T(\mathcal{T})$ by averaging all the degrees of freedom. That means for each interior node $z \in \mathcal{N}(\Omega)$, the derivatives $\partial^{\alpha}\left(J_{1} v_{M}\right)(z)$ is the average of all $\left.\partial^{\alpha} v_{M}\right|_{T}(z)$ for $T \in \mathcal{T}(z)$ and all orders $|\alpha| \leq 1$ and $\partial\left(J_{1} v_{M}\right) / \partial \nu_{E}=\partial v_{M} / \partial \nu_{E}$ at the midpoint $\operatorname{mid}(E)$ for each interior edge $E \in \mathcal{E}(\Omega)$; while the degrees of freedom on the boundary $\partial \Omega$ are set to zero for $J_{1} v_{M} \in H_{0}^{2}(\Omega)$. The edge-bubbles $b_{E, T}:=30\left(\nu_{T} \cdot \nu_{E}\right)$ $\operatorname{dist}\left(z_{3}, E\right) \varphi_{1}^{2} \varphi_{2}^{2} \varphi_{3} \in P_{5}(T)$ (for $T=\operatorname{conv}\left\{z_{1}, z_{2}, z_{3}\right\}=\operatorname{conv}\left\{E, z_{3}\right\} \in \mathcal{T}$ and the nodal basis function $\varphi_{j} \in S^{1}(\mathcal{T})$ associated with $z_{j}$ ) continuously extended by zero for $T \notin \mathcal{T}(E)$ to $b_{E}$ correct the integral mean of the normal derivatives along the edges to guarantee (iii) for $J_{G} v_{M}:=J_{1} v_{M}+\sum_{E \in \mathcal{E}(\Omega)}\left(f_{E}\left(v_{M}-J_{1} v_{M}\right) \mathrm{d} s\right) b_{E}$ [18, Prop. 2.6]. Since the Morley element is continuous in the nodes (i) holds. The characterization of the Morley interpolation operator shows that (i) and (iii) imply (iv). Proposition 2.5 of [18] displays a global version of the estimate (v) (obtained by the sum over $T \in \mathcal{T}$ ); a closer investigation of the proof reveals that the local arguments for the HCT element from [16] (which is almost affine) verify (v). Other $C^{1}$ conforming elements, such as for example the Argyris element, allow for a similar construction of a conforming companion by averaging. The appropriate corrections then guarantee (i) and (iii) and therefore (iv). The choice of $H C T$ in [18] is a natural one because the degrees of freedom fit conveniently to those of the Morley finite element.

\subsection{Proof of $(\mathrm{C} 1)$}

Theorem 3 in [5] asserts $\left\|v_{M}-I_{M} v_{M}\right\|_{L^{2}(K)} \leq \kappa_{M} h_{K}^{2}\left\|D^{2}\left(v_{M}-I_{M} v_{M}\right)\right\|_{L^{2}(K)}$ for all $v \in$ $H^{2}(K)$ and $K \in \mathcal{T}$ with $\kappa_{M}=0.257457844658$. This estimate holds on any coarse and fine triangle $K \in \mathcal{T} \cap \widehat{\mathcal{T}}$. The arguments in $[5,10]$ can be generalized to prove

$$
\left\|\widehat{v}_{M}-I_{M} \widehat{v}_{M}\right\|_{L^{2}(K)} \leq \Lambda_{1} h_{K}^{2}\left\|D^{2}\left(\widehat{v}_{M}-I_{M} \widehat{v}_{M}\right)\right\|_{L^{2}(K)}
$$

for any $K \in \mathcal{T}$ and $\widehat{v}_{M} \in M(\widehat{\mathcal{T}})$ with $\Lambda_{1}$ of $(\mathrm{C} 1)$.

The following soft analysis briefly accounts for $(\mathrm{C} 1)$. Let $K \in \mathcal{T} \backslash \widehat{\mathcal{T}}$ and set $\widehat{w}_{M}:=\left(\widehat{v}_{M}-\right.$ $\left.I_{M} \widehat{v}_{M}\right)\left.\right|_{K}$. It holds $\widehat{w}_{M} \in M^{\prime}(\widehat{\mathcal{T}}(K))$ with the fine triangulation $\widehat{\mathcal{T}}(K):=\{T \in \widehat{\mathcal{T}}: T \subset K\}$ for the domain $\operatorname{int}(K)$ rather than $\Omega, \widehat{w}_{M}(z)=0$ for any node $z \in \mathcal{N}(T)$ and $f_{E} \partial \widehat{w}_{M} / \partial \nu_{T} \mathrm{~d} s=0$ for any edge $E \in \mathcal{E}(T)$. Prop. 2.5-2.6 in [18] allow the definition of a conforming companion operator on the fine triangulation of a coarse triangle, $\widehat{J}: M^{\prime}(\widehat{\mathcal{T}}(K)) \rightarrow H^{2}(K)$ with the properties in Lemma 5.1 for $\widehat{\mathcal{T}}(K)$. Due to the missing boundary conditions in contrast to [18] the gradient in the new boundary nodes $z \in \widehat{\mathcal{N}}(\partial K) \backslash \mathcal{N}(\partial K)$ is computed by averaging over interior triangles

$$
\nabla\left(\widehat{J} \widehat{v}_{M}(z)\right)=|\widehat{\mathcal{T}}(z) \cap \widehat{\mathcal{T}}(K)|^{-1} \sum_{T \in \widehat{\mathcal{T}}(z) \cap \widehat{\mathcal{T}}(K)}\left(\left.\nabla \widehat{v}_{M}\right|_{T}\right)(z)
$$

The triangle inequality reads $\left\|\widehat{w}_{M}\right\|_{L^{2}(K)} \leq\left\|\widehat{w}_{M}-\widehat{J} \widehat{w}_{M}\right\|_{L^{2}(K)}+\left\|\widehat{J} \widehat{w}_{M}\right\|_{L^{2}(K)}$. Lemma 5.1.v proves $\left\|\widehat{w}_{M}-\widehat{J} \widehat{w}_{M}\right\|_{L^{2}(K)} \lesssim h_{K}^{2} \min _{v \in H_{0}^{2}(\Omega)}\left\|D_{\mathrm{NC}}^{2}\left(v_{M}-v\right)\right\|_{L^{2}(K)} \leq h_{K}^{2}\left\|D_{\mathrm{NC}}^{2} v_{M}\right\|_{L^{2}(K)}$ for the first term. Since $I_{M} \widehat{J} \widehat{w}_{M}=0$, the error estimate [5, Thm.3] for the Morley interpolation of $\widehat{J} \widehat{w}_{M} \in H^{2}(K)$ followed by the stability property [18, Prop. 2.6] of the companion operator 
proves

$$
\begin{aligned}
& \left\|\widehat{J} \widehat{w}_{M}\right\|_{L^{2}(K)}=\left\|\widehat{J} \widehat{w}_{M}-I_{M} \widehat{J} \widehat{w}_{M}\right\|_{L^{2}(K)} \leq \kappa_{M} h_{K}^{2}\left\|\left(1-\Pi_{0}\right) D^{2}\left(\widehat{J} \widehat{w}_{M}\right)\right\|_{L^{2}(K)} \\
\leq & \kappa_{M} h_{K}^{2}\left\|D^{2}\left(\widehat{J} \widehat{w}_{M}\right)\right\|_{L^{2}(K)} \leq \kappa_{M} h_{K}^{2}\left(\left\|D_{\mathrm{NC}}^{2}\left(\widehat{J} \widehat{w}_{M}-\widehat{w}_{M}\right)\right\|_{L^{2}(K)}+\left\|D_{\mathrm{NC}}^{2} \widehat{w}_{M}\right\|_{L^{2}(K)}\right) \\
\lesssim & h_{K}^{2}\left\|D_{\mathrm{NC}}^{2} \widehat{w}_{M}\right\|_{L^{2}(K)} .
\end{aligned}
$$

The combination of these estimates shows $\left\|\widehat{w}_{M}\right\|_{L^{2}(K)} \lesssim h_{K}^{2}\left\|D_{\mathrm{NC}}^{2} \widehat{w}_{M}\right\|_{L^{2}(K)}$ for any $K \in \mathcal{T}$. This concludes the proof of $(\mathrm{C} 1)$.

\subsection{Proof of $(\mathrm{C} 2),(\mathrm{C} 5)$}

Since the Hessian $D_{\mathrm{NC}}^{2} w_{M} \in P_{0}\left(\mathcal{T} ; \mathbb{R}^{2 \times 2}\right)$ is piecewise constant for any $w_{M} \in M(\mathcal{T}) \subset$ $P_{2}(\mathcal{T})$, the identity $\Pi_{0} D_{\mathrm{NC}}^{2}=D_{\mathrm{NC}}^{2} I_{M}[5,(3.1)]$ proves $(\mathrm{C} 2)$ by

$$
a_{h}\left(w_{M}, \widehat{v}_{M}-I_{M} \widehat{v}_{M}\right)=\left(D_{\mathrm{NC}}^{2} w_{M},\left(1-\Pi_{0}\right) D_{\mathrm{NC}}^{2} \widehat{v}_{M}\right)_{L^{2}(\Omega)}=0 .
$$

The analogue identity on the refined triangulation $\widehat{\mathcal{T}}$ reads $\widehat{\Pi}_{0} D^{2} v=D_{\mathrm{NC}}^{2} \widehat{I}_{\mathrm{NC}} v$ for all $v \in H_{0}^{2}(\Omega)$. Since $D_{\mathrm{NC}}^{2} w_{M} \in P_{0}\left(\mathcal{T} ; \mathbb{R}^{2 \times 2}\right) \subset P_{0}\left(\widehat{\mathcal{T}} ; \mathbb{R}^{2 \times 2}\right)$, it follows $(\mathrm{C} 5)$ for any $T \in \mathcal{T}$ with $\Lambda_{5}=1$ by $\left\|D_{\mathrm{NC}}^{2}\left(\widehat{I}_{M} v-w_{M}\right)\right\|_{L^{2}(T)}=\left\|\widehat{\Pi}_{0} D^{2}\left(v-w_{M}\right)\right\|_{L^{2}(T)} \leq\left\|D^{2}\left(v-w_{M}\right)\right\|_{L^{2}(T)}$.

\subsection{Proof of $(\mathrm{C} 3)$}

Given any $T \in \mathcal{T} \cap \widehat{\mathcal{T}}$ and some $\widehat{v}_{M} \in M(\widehat{\mathcal{T}})$, it remains to verify that $\left.I_{M} \widehat{v}_{M}\right|_{T}$ and $\left.\widehat{v}_{M}\right|_{T}$ coincide in the degrees of freedom for the Morley finite element. Since $\partial \widehat{v}_{M} /\left.\partial \nu_{E}\right|_{E} \in P_{1}(E)$ implies $\partial \widehat{v}_{M} / \partial \nu_{E}(\operatorname{mid}(E))=f_{E} \partial \widehat{v}_{M} / \partial \nu_{E} \mathrm{~d} s$ for all sides $E \in \mathcal{E}(T)$, the definition of $I_{M}$ shows indeed that the normal derivatives at the edge midpoints $\operatorname{mid}(E)$ for $E \in \mathcal{E}(T)=\widehat{\mathcal{E}}(T)$ and the values in the vertices $z \in \mathcal{N}(T)=\widehat{\mathcal{N}}(T)$ of $\widehat{v}_{M}$ coincide with those of $I_{M} \widehat{v}_{M}$.

\subsection{Proof of $(\mathrm{C} 4)$}

Given any $u_{M} \in M(\mathcal{T})$ and $\widehat{u}_{M}^{*}:=\widehat{I}_{M} J_{G}\left(u_{M}\right)$, Lemma 5.1.iv shows $u_{M}=I_{M} J_{G}\left(u_{M}\right)=$ $I_{M} \widehat{I}_{M} J_{G}\left(u_{M}\right)$. This proves $(\mathrm{C} 4)$.

\subsection{Proof of $(\mathrm{C} 6)$}

Given any $v_{M} \in M(\mathcal{T})$ and $K \in \mathcal{T}$, Lemma 5.1 shows that $J_{G} v_{M}$ and $v_{M}$ have the same nodal values (i) and integral means of the normal derivatives along the edges (iii). Only the derivatives $\nabla\left(J_{G} v_{M}\right)(z)$ for inner nodes $z \in \mathcal{N}(K) \cap \mathcal{N}(\Omega)$ are computed by averaging $\left.\nabla v_{M}\right|_{T}(z)$ for all $T \in \mathcal{T}(z)$ and so $J_{G}$ is associated with local contributions $J_{G, K}$ for any $K \in \mathcal{T}$ in the sense that $\left.J_{G}\left(v_{M}\right)\right|_{K}=J_{G, K}\left(\left.v_{M}\right|_{\Omega(K)}\right)$.

For any $\left.w_{M} \in\left(M(\mathcal{T}) \cap H_{0}^{2}(\Omega)\right)\right|_{\Omega(K)}$, the derivative $\nabla w_{M}$ is continuous in $\Omega(K)$ and vanishes along boundary edges $E \subset \partial \Omega$, hence $\nabla w_{M}(z)=\nabla J_{G} w_{M}(z)$ for all $z \in \mathcal{N}(\Omega(K))$. The nodal values and the integral means of the normal derivatives of $w_{M}$ and $J_{G} w_{M}$ coincide by Lemma 5.1.i and iv. Hence, the functions $\left.\left.w_{M} \in\left(P_{2}(\mathcal{T}) \cap H_{0}^{2}(\Omega)\right)\right|_{\Omega(K)} \subset H C T(\mathcal{T})\right|_{\Omega(K)}$ and $\left.\left.\left.J_{G} w_{M}\right|_{\Omega(K)} \in H C T(\mathcal{T})\right|_{\Omega(K)} \subset H_{0}^{2}(\Omega)\right|_{\Omega(K)}$ coincide in the degrees of freedom for the $H C T$ finite element. Consequently, $\left.w_{M}\right|_{T}=J_{G, T} w_{M}$ for any $T \in \mathcal{T}(\Omega(K))$ proves (C6). 


\subsection{Proof of $(\mathrm{C} 7)$}

The derivative $\nabla_{\mathrm{NC}} v_{M} \in C R_{0}^{1}\left(\mathcal{T} ; \mathbb{R}^{2}\right)$ of a $v_{M} \in M(\mathcal{T})$ is a Crouzeix-Raviart function. Therefore, given any $T \in \mathcal{T}$ and $\left.w_{M} \in M(\mathcal{T})\right|_{\Omega(T)}$, the arguments of Section 4.7 apply for each component of $\nabla_{\mathrm{NC}} w_{M}$ : If $\left\|\left[D_{\mathrm{NC}}^{2} w_{M}\right]_{E} \times \nu_{E}\right\|_{L^{2}(E)}^{2}=0$ for all $E \in \mathcal{E}(\Omega(T))$, then $\nabla w_{M} \in$ $\left.S_{0}^{1}\left(\mathcal{T} ; \mathbb{R}^{2}\right)\right|_{\Omega(T)}$. Consequently, $\left.w_{M} \in\left(M(\mathcal{T}) \cap H_{0}^{2}(\Omega)\right)\right|_{\Omega(T)}$. This proves $(\mathrm{C} 7)$.

\subsection{Towards application in $3 \mathrm{D}$}

The physical application in mind are plate problems, therefore this paper concentrates on the two-dimensional case. However, the Morley element is generalized to solve fourth-order elliptic equations in any space dimension in [21]. Given any $n$-simplex $T \in \mathcal{T}$ with $(n-1)$ dimensional sub-simplices (faces in 3D) $F \in F(\mathcal{T})$ and $(n-2)$-dimensional sub-simplices (sides in 3D) $E \in \mathcal{E}(T)$, [21, Def. 1] introduces the following local $|\mathcal{F}(T)|+|\mathcal{E}(T)|=(n+1)+\left(\begin{array}{c}n+1 \\ n-1\end{array}\right)=$ $(n+1)(n+2) / 2$ degrees of freedom for $v \in P_{2}(\mathcal{T})$

$$
f_{E} v \mathrm{~d} s \quad \text { and } \quad f_{F} \frac{\partial v}{\partial \nu} \mathrm{d} s \quad \text { for all } E \in \mathcal{E}(T), F \in \mathcal{F}(T), \text { and } v \in C^{1}(T) .
$$

If the integral mean over a node $z \in \mathcal{N}$ for $n=2$ is translated as point evaluation, this is a generalization of the two-dimensional definition. In [21, (9)] the dual basis of $M(\mathcal{T})$ in $n=3$ dimension is stated and used to define the standard interpolation $I_{M}: H_{0}^{2}(\Omega)+M(\widehat{\mathcal{T}}) \rightarrow M(\mathcal{T})$ for any $v \in H_{0}^{2}(\Omega)+M(\widehat{\mathcal{T}})$ with

$$
\begin{aligned}
& f_{E} v \mathrm{~d} s=f_{E} I_{M} v \mathrm{~d} s \quad \text { for any } E \in \mathcal{E} \quad \text { and } \\
& f_{F} \frac{\partial I_{M}(v)}{\partial \nu_{F}} \mathrm{~d} s=\frac{\partial I_{M} v}{\partial \nu_{F}}(\operatorname{mid}(F))=f_{F} \frac{\partial v}{\partial \nu_{F}} \mathrm{~d} s \quad \text { for any } F \in \mathcal{F} .
\end{aligned}
$$

An integration by parts proves $\Pi_{0} D^{2}=D_{N C}^{2} I_{M}$ which leads to (C2) and (C5) as in 2D. The condition (C3) holds with the same arguments as in Section 5.4, while (C1) remains to be discussed. However, [10, Thm. 3.5] for the Crouzeix-Raviart case holds in any space dimension and the gradient $\nabla_{\mathrm{NC}} v_{M} \in C R^{1}\left(\mathcal{T} ; \mathbb{R}^{n}\right)$ is a Crouzeix-Raviart function in $n$ components for any $v_{m} \in M(\mathcal{T})$, hence the authors are optimistic that the proof of $(\mathrm{C} 1)$ carries over to higher space dimension. Moreover, since Section 4.7 holds for all $n \in \mathbb{N}$, (C7) follows as above.

To verify the conditions $(\mathrm{C} 4)$ and $(\mathrm{C} 6)$ a $C^{1}$-conforming space in higher dimension has to be chosen. In [26] a composite $C^{1}$ tetrahedral element $W(\mathcal{T})$ is presented. Thereby each tetrahedron $T \in \mathcal{T}$ is subdivided into four tetrahedra $T_{F}:=\operatorname{conv}\{F, \operatorname{mid}(T)\} \in \mathcal{K}(T)$ with the following 45 degrees of freedom, for any $v \in W(T)$,

(1) $v(z), \nabla v(z)$ and $D^{2} v(z)$ at the four vertices $z \in \mathcal{N}(T)$,

(2) $\nabla v(\operatorname{mid}(F)) \cdot \nu_{F}$ at the midpoints of the four faces $F \in \mathcal{F}(T)$,

(3) $v(\operatorname{mid}(T))$ at the centroid $\operatorname{mid}(T)$,

where $v \in P^{5}(\mathcal{K}(T)) \cap C^{1}(T) \cap C^{4}(\operatorname{mid}(T))$ is a piecewise $P_{5}$ element and the normal derivatives on the faces $\nabla v \cdot \nu_{F} \in P_{3}(F)$ are constrained to be cubic along each $F \in \mathcal{F}$. The interpolation operator $J_{1}: M(\mathcal{T}) \rightarrow W(\mathcal{T})$ is defined by averaging as follows. Given any $v_{M} \in \mathcal{M}(\mathcal{T})$ define the degrees of freedom for $W(\mathcal{T})$ by $J_{1} D^{\alpha} v_{M}(z)=\left.|\mathcal{T}(z)|^{-1} \sum_{T \in \mathcal{T}(z)} D^{\alpha} v_{M}\right|_{T}(z)$ for all $z \in \mathcal{N}(\Omega)$ and $0 \leq|\alpha| \leq 1$ and zero otherwise, set $\nabla J_{1} v_{M}(\operatorname{mid}(F)) \cdot \nu_{F}=\nabla v_{M}(\operatorname{mid}(F)) \cdot \nu_{F}$ 
for all $F \in \mathcal{F}$, and $J_{1} v_{M}(\operatorname{mid}(T))=v_{M}(\operatorname{mid}(T))$ for all $T \in \mathcal{T}$. This companion (with the local corrections indicated below) satisfies the localisation condition $(\mathrm{C} 6)$. To verify $(\mathrm{C} 4)$ for $\widehat{u}_{M}^{*}=\widehat{I}_{M} J_{2}\left(u_{M}\right)$ a condition comparable to Lemma 5.1.iv would suffice. Therefore, the integral means of the function along the edges and of the normal derivatives along the faces have to be corrected without changing any of the degrees of freedom in $W(\mathcal{T})$. Due to the degree of freedom in the midpoint of each simplex a refined triangulation is introduced. Let $\mathcal{K} \in \mathbb{T}(\mathcal{T})$ denote the refinement, where each tetrahedra is divided in four sub-tetrahedra with the centroid as new vertex, i.e., $\mathcal{K}:=\bigcup_{T \in \mathcal{T}} \bigcup_{T_{F} \in \mathcal{K}(T)} T_{F}$. For any $E \in \mathcal{E}(\Omega)$ chose a function $\xi_{E} \in H_{0}^{2}(\Omega)$ with $f_{G} \xi_{E} \mathrm{~d} s=\delta_{G E}$ for all $G \in \mathcal{E}$, such that $\operatorname{supp}\left(\xi_{E}\right) \subset \widehat{\omega}_{E}:=\operatorname{int}\left(\bigcup_{K \in \mathcal{K}(E)} K\right)$ and $\nabla \xi_{E} \cdot \nu_{F}(\operatorname{mid}(F))=0$ for all sides $F \in \mathcal{F}$. Since there exists $0<\varepsilon<\min _{F \in \mathcal{F}} h_{F} / 2$ with $B_{\varepsilon}(\operatorname{mid}(E)) \subset \widehat{\omega}_{E}$, a possible choice is a mollifier $\xi_{E} \in C^{\infty}\left(\mathbb{R}^{3}\right)$ with $\operatorname{supp}\left(\xi_{E}\right) \subset B_{\varepsilon}(\operatorname{mid}(E))$ such that without loss of generality $f_{E} \xi_{E} \mathrm{~d} s=1$. There are also higher-order conforming polynomials that could be chosen for this correction. For example in [27, Cor. 2.1] a $C^{1}$ conforming element in $P_{k}(\mathcal{T})$ for $9 \leq k$ is introduced. For $k=10$ this element has one interior point of each edge $E \in \mathcal{E}$ as degree of freedom. The associated dual basis function $\xi_{E} \in P_{10}(\mathcal{K}) \cap C^{1}(\Omega)$ normalized such that $f_{E} \xi_{E} \mathrm{~d} s=1$ is an other possible choice. For any $v_{M} \in M(\mathcal{T})$ set

$$
\tilde{J}_{1}\left(v_{M}\right)=J_{1} v_{M}+\sum_{E \in \mathcal{E}}\left(f_{E}\left(v_{M}-J_{1} v_{M}\right) \mathrm{d} s\right) \xi_{E} \in C^{1}(\Omega) .
$$

For the correction of the integral mean of the normal derivatives along the faces choose for each $F \in \mathcal{F}(\Omega)$ a function $\zeta_{F} \in H_{0}^{2}(\Omega)$ with $\operatorname{supp}\left(\zeta_{F}\right) \subset \widehat{\omega}_{F}:=\operatorname{int}\left(\bigcup_{K \in \mathcal{K}(F)} K\right)$ and $f_{G} \nabla \zeta_{F} \cdot \nu_{F} \mathrm{~d} s=$ $\delta_{G F}$ for all $G \in \mathcal{F}$. Inspired by [18] a piecewise polynomial $\zeta_{F} \in P_{7}(\mathcal{K}) \cap C^{1}(\Omega)$ with this attributes comes to mind. For any $v_{M} \in M(\mathcal{T})$ set

$$
J_{2}\left(v_{M}\right):=\tilde{J}_{1} v_{M}+\sum_{F \in \mathcal{F}}\left(f_{F} \nabla\left(v_{M}-\tilde{J}_{1} v_{M}\right) \cdot \nu_{F} \mathrm{~d} s\right) \zeta_{F} \in C^{1}(\Omega) .
$$

By construction holds $I_{M} J_{2} v_{M}=v_{M}$ for any Morley function $v_{M} \in M(\mathcal{T})$, which is the missing equality in the proof of the remaining condition $(\mathrm{C} 4)$.

Other $C^{1}$-conforming elements, such as for example the element in [27] allow for a similar construction of the conforming companion $J_{2}$ by averaging (and perhaps appropriate corrections).

\section{Refined Analysis}

This section introduces the piecewise design of companion operators in Section 6.1-6.2 based on a fixed subset of sides $\mathcal{F}^{\prime} \subseteq \mathcal{F}$. This leads in Section 6.3 to the definition of an alternative approximation $\widehat{u}_{h}^{*} \in V(\widehat{\mathcal{T}})$ to the discrete solution $u_{h} \in V(\mathcal{T})$ in (1.3) with $(\mathrm{C} 4)$ and to (1.4) with $\mathcal{T} \backslash \widehat{\mathcal{T}}$ replacing $\mathcal{R}$ for Crouzeix-Raviart and Morley finite element methods. A closer look reveals that merely the jump contribution along coarse-but-not-fine sides $\mathcal{F} \backslash \widehat{\mathcal{F}}$ occur in (1.4); in fact,

$$
\left\|\widehat{u}_{h}^{*}-u_{h}\right\|_{h}^{2} \lesssim \sum_{F \in \mathcal{F} \backslash \widehat{\mathcal{F}}} h_{F}\left\|\left[D^{m} u_{h}\right]_{F} \times \nu_{F}\right\|_{L^{2}(F)}^{2} .
$$

The remaining conditions (C1)-(C3) sufficient for (1.3) depend only on the interpolation operators in Sections 4 and 5, so that (6.1) implies the discrete reliability (dRel) with $\mathcal{T} \backslash \widehat{\mathcal{T}}$ replacing $\mathcal{R}$. 


\subsection{Piecewise companion operator for piecewise affines}

The piecewise design of a companion operator is based on a set of sides $\mathcal{F}^{\prime} \subseteq \mathcal{F}$ and its associated sets $\mathcal{T}(K, z)$ and $\mathcal{F}(K, z)$ for any simplex $K \in \mathcal{T}$ with vertex $z \in \mathcal{N}(K)$ in the sequel. Recall the set $\mathcal{T}(z):=\{T \in \mathcal{T}: z \in \mathcal{N}(T)\}$ of simplices with vertex $z \in \mathcal{N}$ and the set $\mathcal{F}(z):=\{F \in \mathcal{F}: z \in \mathcal{N}(F)\}$ of sides with vertex $z \in \mathcal{N}$.

Definition 6.1. Given $\mathcal{F}^{\prime} \subseteq \mathcal{F}$, a simplex $K \in \mathcal{T}$, and its vertex $z \in \mathcal{N}(K)$, let

$$
\begin{aligned}
& \mathcal{T}(K, z):=\left\{T \in \mathcal{T}: \text { there exist } T_{1}, \ldots, T_{J} \in \mathcal{T}(z) \text { with } T_{1}=K, T_{J}=T,\right. \\
& \text { and } \left.\partial T_{j} \cap \partial T_{j+1} \in \mathcal{F}^{\prime} \text { for all } j=1, \ldots, J-1\right\} \subseteq \mathcal{T}(z)
\end{aligned}
$$

denote the side-connectivity component with respect to $\mathcal{F}^{\prime}$ of $K$ in $\mathcal{T}(z)$ with cardinality $|\mathcal{T}(K, z)|$. Under the same premise let

$$
\mathcal{F}(K, z):=\mathcal{F}^{\prime} \cap\left\{F \in \mathcal{F}(z): F \in \partial T_{1} \cap \partial T_{2} \text { for } T_{1}, T_{2} \in \mathcal{T}(K, z)\right\}
$$

denote the set of interior edges in $\mathcal{T}(K, z)$. Abbreviate $\mathcal{F}^{\prime}(\partial \Omega):=\mathcal{F}^{\prime} \cap \mathcal{F}(\partial \Omega)$ for the set of boundary sides in $\mathcal{F}^{\prime}$. (Notice $K \in \mathcal{T}(K, z)$ for any $K \in \mathcal{T}, z \in \mathcal{N}(K)$.)

There are two extreme examples for the choice of $\mathcal{F}^{\prime}$ and the applications below concern some intermediate selection in Section 6.3 illustrated in Fig. 6.1.

Example 6.1. (a) The maximal set $\mathcal{F}^{\prime}=\mathcal{F}$ means $\mathcal{T}(K, z)=\mathcal{T}(z)$ for any $K \in \mathcal{T}$ and $z \in \mathcal{N}(K)$. This choice in Definition 6.2-6.3 leads to the conforming companion operator of (4.2). In Definition 6.4-6.5 it leads to $J_{G}$ in Lemma 5.1.

(b) If $\mathcal{F}^{\prime} \cap \mathcal{F}(z) \cap \mathcal{F}(K)=\emptyset$ for $K \in \mathcal{T}$ and $z \in \mathcal{N}(K)$, then $\{K\}=\mathcal{T}(K, z)$ (the condition $\partial T_{j} \cap \partial T_{j+1} \in \mathcal{F}^{\prime}$ for $j=1, \ldots, J-1$ does not arise for $J=1$ ); singletons are side connected.

Any choice of $\mathcal{F}^{\prime} \subseteq \mathcal{F}$ allows the definition of the local companion operator below for CrouzeixRaviart in Definition 6.2-6.3 $(k=m=1, n \geq 2)$ and for the Morley finite element in Definition 6.4-6.5 $(k=m=2=n)$.

Definition 6.2 (Local companion $\boldsymbol{J}_{\mathbf{1}}$ for piecewise affines). Suppose $\mathcal{T} \in \mathbb{T}$ and the sets $\mathcal{T}(K, z)$ are as in Definition 6.1 associated with $\mathcal{F}^{\prime} \subseteq \mathcal{F}$ to define $J_{1}: P_{1}(\mathcal{T}) \rightarrow P_{1}(\mathcal{T})$ as follows. For any $v_{1} \in P_{1}(\mathcal{T})$ and $K \in \mathcal{T}$ define $\left.\left(J_{1} v_{1}\right)\right|_{K} \in P_{1}(K)$ through linear interpolation in $K$ of the nodal values

$$
\left.\left(J_{1} v_{1}\right)\right|_{K}(z):=\left\{\begin{array}{l}
0, \quad \text { if } z \in \mathcal{N}(F) \text { for some } F \in \mathcal{F}(K) \cap \mathcal{F}^{\prime}(\partial \Omega), \\
\left.|\mathcal{T}(K, z)|^{-1} \sum_{T \in \mathcal{T}(K, z)} v_{1}\right|_{T}(z), \quad \text { else }
\end{array}\right.
$$

at the $n+1$ vertices $z \in \mathcal{N}(K)$ of $K$.

The values at e.g. interior vertices are computed by averaging over the side-connected $\mathcal{T}(K, z) \subseteq$ $\mathcal{T}(z)$ of cardinality $|\mathcal{T}(K, z)|$. The first alternative in (6.3) at all vertices of a boundary side $F \in$ $\mathcal{F}^{\prime}(\partial \Omega)$ enforces homogeneous boundary conditions. The piecewise affine $J_{1} v_{1}$ is discontinuous and violates homogeneous boundary conditions in general. For $n \geq 2$ the normalized sidebubbles

$$
b_{F}:=\left(\prod_{z \in \mathcal{N}(F)} \varphi_{z}\right) / f_{F}\left(\prod_{z \in \mathcal{N}(F)} \varphi_{z}\right) \mathrm{d} s \in S^{n}(\mathcal{T}) \quad \text { for } F \in \mathcal{F}
$$


utilize the nodal basis-function $\varphi_{z} \in S^{1}(\mathcal{T})=P_{1}(\mathcal{T}) \cap C(\bar{\Omega})$ associated to $z \in \mathcal{N}$. The subsequent correction assures that the operator $J_{2}: P_{1}(\mathcal{T}) \rightarrow P_{n}(\mathcal{T})$ preserves the integral means of $v_{1} \in P_{1}(\mathcal{T})$ along all sides $F \in \mathcal{F}$.

Definition 6.3 (Local companion $\boldsymbol{J}_{\boldsymbol{n}}$ for piecewise affines). For any $K \in \mathcal{T}, v_{1} \in P_{1}(\mathcal{T})$, and $J_{1} v_{1}$ of Definition 6.2 , set

$$
\left.\left(J_{n} v_{1}\right)\right|_{K}:=\left.\left(J_{1} v_{1}\right)\right|_{K}+\left.\sum_{F \in \mathcal{F}(K)}\left(\left.f_{F}\left(v_{1}-J_{1} v_{1}\right)\right|_{K} \mathrm{~d} s\right) b_{F}\right|_{K} \in P_{n}(K) .
$$

The following properties of the companion operators from Definition $6.2-6.3$ will be employed throughout this section.

Lemma 6.1 (Properties of $\boldsymbol{J}_{\mathbf{1}}, \boldsymbol{J}_{\boldsymbol{n}}$ for piecewise affines). $\quad$ (a) Given any $v_{1} \in P_{1}(\mathcal{T})$, the jump $\left[J_{1} v_{1}\right]_{F}=0$ of $J_{1} v_{1}$ vanishes along any $F \in \mathcal{F}^{\prime}$. In particular, the companion $\left.J_{1} v_{1}\right|_{\omega_{F}} \in S^{1}(\mathcal{T}(F))$ is continuous along any $F \in \mathcal{F}^{\prime} \cap \mathcal{F}(\Omega)$ and vanishes along $F \in \mathcal{F}^{\prime}(\partial \Omega)$.

(b) Given any $v_{1} \in P_{1}(\mathcal{T})$, the companion $J_{n} v_{1}$ preserves the integral mean $\left.f_{F}\left(J_{n} v_{1}\right)\right|_{K} \mathrm{~d} s=$ $\left.f_{F} v_{1}\right|_{K} \mathrm{~d} s$ along any side $F \in \mathcal{F}(K)$ in $K \in \mathcal{T}$ and the jump $\left[J_{n} v_{1}\right]_{F}=0$ vanishes along $F \in \mathcal{F}^{\prime}$.

(c) If a simplex $K \in \mathcal{T}$ is isolated in the sense that $\mathcal{F}(K) \cap \mathcal{F}^{\prime}=\emptyset$, Definition 6.2-6.3 imply $\left.v_{1}\right|_{K}=\left.\left(J_{1} v_{1}\right)\right|_{K}=\left.\left(J_{n} v_{1}\right)\right|_{K}$ for all $v_{1} \in P_{1}(\mathcal{T})$.

Proof of (a). For any interior side $F \in \mathcal{F}^{\prime} \cap \mathcal{F}(\Omega)$ with side-patch $\omega_{F}=\operatorname{int}\left(T_{+} \cup T_{-}\right)$and $\mathcal{T}(F):=\left\{T_{+}, T_{-}\right\}$, Definition 6.1 implies $\mathcal{T}\left(T_{+}, z\right)=\mathcal{T}\left(T_{-}, z\right)$ for any vertex $z \in \mathcal{N}(F)$. Hence, Definition 6.2 shows $\left.\left(J_{1} v_{1}\right)\right|_{T_{+}}(z)=\left.\left(J_{1} v_{1}\right)\right|_{T_{-}}(z)$ for all $z \in \mathcal{N}(F)$. Along any boundary side $F \in \mathcal{F}^{\prime}(\partial \Omega),\left.J_{1} v_{1}\right|_{F}=0$ vanishes by Definition 6.2. This proves (a).

Proof of (b). Since $f_{E} b_{F} \mathrm{~d} s=\delta_{E F}$ and $\operatorname{supp}\left(b_{F}\right)=\omega_{F}$ for any $E, F \in \mathcal{F}$, the operator $J_{n}$ in Definition 6.3 preserves the integral means. The continuity of $b_{F} \in C(\Omega)$ and (a) imply $\left[J_{n} v_{1}\right]_{F}=0$ for any $F \in \mathcal{F}^{\prime}$.

Proof of (c). This is elementary for $\mathcal{T}(K, z)=\{K\}$ for all $K \in \mathcal{T}$ with $\mathcal{F}(K) \cap \mathcal{F}^{\prime}=\emptyset$.

The following theorem provides a local a posteriori approximation error estimate for the operator $J_{n}$ of Definition 6.3; recall $\mathcal{F}^{\prime}(\partial \Omega):=\mathcal{F}^{\prime} \cap \mathcal{F}(\partial \Omega)$ and $\mathcal{F}(K, z):=\mathcal{F}^{\prime} \cap\{F \in \mathcal{F}(z): F \in$ $\partial T_{1} \cap \partial T_{2}$ for $\left.T_{1}, T_{2} \in \mathcal{T}(K, z)\right\}$ from Definition 6.1.

Theorem 6.1 (Approximation error). Given (A1), $K \in \mathcal{T}$, and $v_{1} \in P_{1}(\mathcal{T})$, the companion $J_{n} v_{1}$ of Definition 6.3 satisfies

$$
C_{n}^{-1} h_{K}^{-1}\left\|v_{1}-J_{n} v_{1}\right\|_{L^{2}(K)}^{2} \leq \sum_{z \in \mathcal{N}(K)} \sum_{F \in \mathcal{F}(K, z)}\left\|\left[v_{1}\right]_{F}\right\|_{L^{2}(F)}^{2}+\sum_{F \in \mathcal{F}(K) \cap \mathcal{F}^{\prime}(\partial \Omega)}\left\|v_{1}\right\|_{L^{2}(F)}^{2} .
$$

The constant $C_{n}$ soley depends on $n$ and $M_{2}$ from (A1).

Proof. Step 1. Definition 6.3 and the triangle inequality show

$$
\left\|v_{1}-J_{n} v_{1}\right\|_{L^{2}(K)} \leq\left\|v_{1}-J_{1} v_{1}\right\|_{L^{2}(K)}+\left\|\sum_{F \in \mathcal{F}(K)}\left|f_{F}\left(v_{1}-J_{1} v_{1}\right)\right|_{K} \mathrm{~d} s \mid b_{F}\right\|_{L^{2}(K)} .
$$


The local mass matrix for normalized bubble functions in $K \in \mathcal{T}$ is SPD and reads

$$
B(K):=\left(\int_{K} b_{E} b_{F} \mathrm{~d} x\right)_{E, F \in \mathcal{F}(K)}=\left(\frac{2^{n-3}((2 n) !)^{2}|K|}{(3 n) ! n !}\left(1+\delta_{E F}\right)\right)_{E, F \in \mathcal{F}(K)} \in \mathbb{R}^{(n+1) \times(n+1)} .
$$

It has the multiple eigenvalue $\lambda_{\min }:=2^{n-3}((2 n) !)^{2}|K| /((3 n) ! n !)$ and the simple eigenvalue $\lambda_{\max }:=(n+2) \lambda_{\min }$. This proves

$$
\left\|\left.\left.\sum_{F \in \mathcal{F}(K)}\left|f_{F}\left(v_{1}-J_{1} v_{1}\right)\right|_{K} \mathrm{~d} s\left|b_{F} \|_{L^{2}(K)}^{2} \leq \lambda_{\max } \sum_{F \in \mathcal{F}(K)}\right| f_{F}\left(v_{1}-J_{1} v_{1}\right)\right|_{K} \mathrm{~d} s\right|^{2} .\right.
$$

Lemma $\mathrm{D}$ of the appendix quantifies the constant in the discrete trace inequality and implies

$$
\left.\sum_{F \in \mathcal{F}(K)}\left|f_{F}\left(v_{1}-J_{1} v_{1}\right)\right|_{K} \mathrm{~d} s\right|^{2} \leq \frac{(n+1)}{|K|}\left\|v_{1}-J_{1} v_{1}\right\|_{L^{2}(K)}^{2} .
$$

Consequently, the constant $C_{J}:=1+(2 n) ! \sqrt{\frac{2^{n-3}(n+1)(n+2)}{(3 n) ! n !}}$ satisfies

$$
\left\|v_{1}-J_{n} v_{1}\right\|_{L^{2}(K)} \leq C_{J}\left\|v_{1}-J_{1} v_{1}\right\|_{L^{2}(K)} .
$$

(It holds $C_{J}=1+2 \sqrt{3 / 5} \leq 2.5492$ for $n=2$ and $C_{J}=1+10 / \sqrt{21} \leq 3.1822$ for $n=3$.)

Step 2. For any $z \in \mathcal{N}(K)$ set $e_{K}(z):=\left.\left(v_{1}-J_{1} v_{1}\right)\right|_{K}(z)$ with the associated coefficient vector $e_{K}:=\left(e_{K}(z)\right)_{z \in \mathcal{N}(K)} \in \mathbb{R}^{n+1}$. The local mass matrix for the $P_{1}$-conforming FEM is $\mathrm{SPD}$ and reads

$$
M(K)=\left(\frac{|K|\left(1+\delta_{j k}\right)}{(n+1)(n+2)}\right)_{j, k=1, \ldots, n+1} \in \mathbb{R}^{(n+1) \times(n+1)} .
$$

The simple eigenvalue $|K| /(n+1)$ of $M(K)$ has the eigenvector $(1, \ldots, 1) \in \mathbb{R}^{n+1}$. The eigenvalue $|K| /((n+1)(n+2))$ has the $n$-dimensional eigenspace of vectors in $\mathbb{R}^{n+1}$ perpendicular to $(1, \ldots, 1)$. Therefore, the affine function $\left.\left(v_{1}-J_{1} v_{1}\right)\right|_{K} \in P_{1}(K)$ satisfies

$$
\left\|v_{1}-J_{1} v_{1}\right\|_{L^{2}(K)}^{2}=e_{K} \cdot M(K) e_{K} \leq \frac{|K|}{n+1} \sum_{z \in \mathcal{N}(K)} e_{K}(z)^{2}
$$

Step 3. Given $z \in \mathcal{N}(K)$ with $\left.\left(J_{1} v_{1}\right)\right|_{K}(z):=\left.j^{-1} \sum_{T \in \mathcal{T}(K, z)} v_{1}\right|_{T}(z)$ for $j=|\mathcal{T}(K, z)| \leq$ $M_{2}$. Choose an enumeration $\left\{T_{1}, \ldots, T_{j}\right\}$ of $\mathcal{T}(K, z)$ such that the values $x_{k}:=\left.\left(J_{1} v_{1}\right)\right|_{K}(z)-$ $\left.v_{1}\right|_{T_{k}}(z) \in \mathbb{R}$ for $k=1, \ldots, j$ are ordered in the sense that $x_{1} \leq x_{2} \leq \cdots \leq x_{j}$. The definition of $\left.\left(J_{1} v_{1}\right)\right|_{K}(z)$ guarantees that the sum $\sum_{k=1}^{j} x_{k}=0$ vanishes. In an abstract notation, Lemma $\mathrm{C}$ of the appendix implies the last inequality (with the displayed constant) in

$$
\begin{aligned}
\left|e_{K}(z)\right|^{2} & \leq \max _{T \in \mathcal{T}(K, z)}\left|v_{1}\right|_{T}(z)-\left.\left.\left(J_{1} v_{1}\right)\right|_{K}(z)\right|^{2} \\
& =\max _{1 \leq k \leq j}\left|x_{k}\right|^{2} \leq \frac{(j-1)(2 j-1)}{6 j} \sum_{k=1}^{j-1}\left|x_{k+1}-x_{k}\right|^{2} .
\end{aligned}
$$

Let $\mathcal{J}:=\left\{\{\alpha, \beta\}: T_{\alpha}, T_{\beta} \in \mathcal{T}(K, z)\right.$ and $\left.\partial T_{\alpha} \cap \partial T_{\beta} \in \mathcal{F}^{\prime}\right\}$ denote the set of unordered index pairs of all simplices in $\mathcal{T}(K, z)$ which share as side in $\mathcal{F}^{\prime}$. The choice of $\mathcal{T}(K, z)$ in (6.2) implies 
that $\mathcal{J}$ is connected, in the sense that for all $\alpha, \beta \in\{1, \ldots, j\}$ and $\alpha \neq \beta$ there are $k \in \mathbb{N}$ pairs $\left\{\alpha_{1}, \alpha_{2}\right\},\left\{\alpha_{2}, \alpha_{3}\right\}, \ldots,\left\{\alpha_{k}, \alpha_{k+1}\right\} \in \mathcal{J}$ with $\alpha_{1}=\alpha$ and $\alpha_{k+1}=\beta$. Lemma B of the appendix implies the first inequality in

$$
\begin{aligned}
& \sum_{k=1}^{j-1}\left|x_{k+1}-x_{k}\right|^{2} \leq \sum_{\{\alpha, \beta\} \in \mathcal{J}}\left|x_{\alpha}-x_{\beta}\right|^{2} \\
= & \sum_{\{\alpha, \beta\} \in \mathcal{J}}\left|\left(\left.v_{1}\right|_{T_{\alpha}}\right)(z)-\left(\left.v_{1}\right|_{T_{\beta}}\right)(z)\right|^{2}=\sum_{F \in \mathcal{F}(K, z)}\left|\left[v_{1}\right]_{F}(z)\right|^{2} .
\end{aligned}
$$

Consequently, $\left|e_{K}(z)\right|^{2} \leq(j-1)(2 j-1) /(6 j) \sum_{F \in \mathcal{F}(K, z)}\left|\left[v_{1}\right]_{F}(z)\right|^{2}$. Note, $j=|\mathcal{T}(K, z)| \leq M_{2}$ is uniformly bounded for any $K \in \mathcal{T} \in \mathbb{T}, z \in \mathcal{N}(K)$.

Step 4. If $z \in \mathcal{N}(K) \cap \mathcal{N}(\partial \Omega)$ belongs to a boundary side $F \in \mathcal{F}(K) \cap \mathcal{F}^{\prime}(\partial \Omega)$ and $\left.\left(J_{1} v_{1}\right)\right|_{K}(z):=0$, the jump definition guarantees $\left|e_{K}(z)\right|=\left|v_{1}\right|_{K}(z)|=|\left[v_{1}\right]_{F}(z) \mid$.

Step 5. Corollary D of the appendix provides the estimate $|F|\left|\left[v_{1}\right]_{F}(z)\right|^{2} \leq n^{2}\left\|\left[v_{1}\right]_{F}\right\|_{L^{2}(F)}^{2}$ for $\left[v_{1}\right]_{F} \in P_{1}(F)$ on any side $F \in \mathcal{F}$ with vertex $z \in \mathcal{N}(F)$.

Step 6. Set $M:=\max \left\{n,\left(M_{2}-1\right)\left(2 M_{2}-1\right) /\left(6 M_{2}\right)\right\}$. The combination of Steps $1-5$ shows

$$
\begin{aligned}
& \left\|v_{1}-J_{n} v_{1}\right\|_{L^{2}(K)}^{2} \\
\leq & \frac{C_{J}^{2} M n^{2}|K|}{n+1}\left(\sum_{z \in \mathcal{N}(K)} \sum_{F \in \mathcal{F}(K, z)} \frac{\left\|\left[v_{1}\right]_{F}\right\|_{L^{2}(F)}^{2}}{|F|}+\sum_{F \in \mathcal{F}(K) \cap \mathcal{F}^{\prime}(\partial \Omega)} \frac{\left\|\left[v_{1}\right]_{F}\right\|_{L^{2}(F)}^{2}}{|F|}\right) .
\end{aligned}
$$

Let $\varrho_{F}=n|K| /|F| \leq h_{K}$ be the height of the vertex $P_{F}$ opposite to the side $F$ in the simplex $K=\operatorname{conv}\left\{F, P_{F}\right\}$. This proves the theorem with $C_{n}:=C_{J}^{2} M n /(n+1)$.

Remark 6.1 $\left(C_{n}\right.$ for Crouzeix-Raviart). For any Crouzeix-Raviart function $v_{C R} \in C R_{0}^{1}(\mathcal{T})$ the integral mean of the jump $f_{F}\left[v_{C R}\right]_{F} \mathrm{~d} s=0$ vanishes along any side $F \in \mathcal{F}$ of diameter $h_{F}:=\operatorname{diam}(F) \leq h_{K}$. The Poincaré inequality (with Payne-Weinberger constant) implies

$$
\left\|\left[v_{C R}\right]_{F}\right\|_{L^{2}(F)}=\left\|\left[v_{C R}\right]_{F}-f_{F}\left[v_{C R}\right]_{F} \mathrm{~d} s\right\|_{L^{2}(F)} \leq h_{F} \pi^{-1}\left\|\left[\nabla_{N C} v_{C R}\right]_{F} \times \nu_{F}\right\|_{L^{2}(F)} .
$$

This and Theorem 6.1 show for any $K \in \mathcal{T}, v_{C R} \in C R_{0}^{1}(\mathcal{T})$, the companion $\left.\left(J_{n} v_{C R}\right)\right|_{K}$ from Definition 6.3 , and $C_{n}^{\prime}:=C_{n} / \pi^{2}$ that

$$
\begin{aligned}
& C^{\prime-1} h_{K}^{-2}\left\|v_{C R}-J_{n} v_{C R}\right\|_{L^{2}(K)}^{2} \\
\leq & \sum_{z \in \mathcal{N}(K)} \sum_{F \in \mathcal{F}(K, z)} h_{F}\left\|\left[\nabla_{N C} v_{C R}\right]_{F} \times \nu_{F}\right\|_{L^{2}(F)}^{2}+\sum_{F \in \mathcal{F}(K) \cap \mathcal{F}^{\prime}(\partial \Omega)} h_{F}\left\|\left[\nabla_{N C} v_{C R}\right]_{F} \times \nu_{F}\right\|_{L^{2}(F)}^{2} .
\end{aligned}
$$

The continuity of $v_{C R} \in C R_{0}^{1}(\mathcal{T})$ in face midpoints guarantees for each $F \in \mathcal{F}$ that the jump $\left[v_{C R}\right]_{F}(x)=\left[\nabla_{\mathrm{NC}} v_{C R}\right]_{F} \cdot(x-\operatorname{mid}(F))$ at $x \in F$. The orthogonality $(z-\operatorname{mid}(F)) \cdot \nu_{F}=0$ and $|z-\operatorname{mid}(F)| \leq h_{F}(n-1) / n$ for all $z \in \mathcal{N}(F)$ result in

$$
\begin{aligned}
\left|\left[v_{C R}\right]_{F}(z)\right| & \leq\left|\left[\nabla_{\mathrm{NC}} v_{C R}\right]_{F} \times \nu_{F}\right||z-\operatorname{mid}(F)| \leq \frac{n-1}{n} h_{F}\left|\left[\nabla_{\mathrm{NC}} v_{C R}\right]_{F} \times \nu_{F}\right| \\
& =\frac{(n-1) h_{F}}{n \sqrt{|F|}}\left\|\left[\nabla_{\mathrm{NC}} v_{C R}\right]_{F} \times \nu_{F}\right\|_{L^{2}(F)} .
\end{aligned}
$$


This replaces Step 5 in the proof of Theorem 6.1 and so leads to (6.5) with

$$
C_{n}^{\prime}:=C_{J}^{2} M \frac{(n-1)^{2}}{n^{3}(n+1)}<\frac{C_{n}}{\pi^{2}}
$$

For $n=2, C_{2}^{\prime} \leq(1+2 \sqrt{3 / 5})^{2} \max \left\{2,\left(M_{2}-1\right)\left(2 M_{2}-1\right) /\left(6 M_{2}\right)\right\} / 24$ (and $C_{2}^{\prime} \leq 0.5924$ for a triangulation in right isosceles triangles or more general with $\left.M_{2} \leq 8\right)$.

\subsection{Piecewise companion operator for piecewise quadratics}

In the case of piecewise quadratic polynomials we restrict the analysis to $n=2$, where $\mathcal{T} \in \mathbb{T}$ is a regular triangulation of $\Omega \subset \mathbb{R}^{2}$ into triangles and let $\mathcal{E}$ denote the set of all edges (rather than writing $\mathcal{F} \equiv \mathcal{E}$ in $2 \mathrm{D}$ ). The local version of the HCT finite element space in (5.1) without boundary conditions reads

$$
H C T^{\prime}(K):=H C T^{\prime}(\{K\}):=\left\{v \in H^{2}(K): v \in P_{3}(\mathcal{K}(K))\right\} \quad \text { for any } K \in \mathcal{T} .
$$

Definition 6.4 (Local companion $\boldsymbol{J}_{\mathbf{1}}$ for piecewise quadratics). Suppose $\mathcal{T} \in \mathbb{T}$ and $\mathcal{T}(K, z)$ associated with $\mathcal{E}^{\prime} \subseteq \mathcal{E}$ as in Definition 6.1. Define $J_{1}: P_{2}(\mathcal{T}) \rightarrow \prod_{K \in \mathcal{T}} H C T^{\prime}(K)$ as follows. For any $v_{2} \in P_{2}(\mathcal{T})$ and any triangle $K \in \mathcal{T}$ define $\left.\left(J_{1} v_{2}\right)\right|_{K} \in H C T^{\prime}(K)$ through the HCT interpolation of the degrees of freedom at the three midpoints $\operatorname{mid}(E)$ of the edges $E \in \mathcal{E}(K)$ and the three vertices $z \in \mathcal{N}(K)$ of $K$ by

$$
\begin{aligned}
& \frac{\left.\partial\left(J_{1} v_{2}\right)\right|_{K}}{\partial \nu_{E}}(\operatorname{mid}(E))=\frac{\left.\partial v_{2}\right|_{K}}{\partial \nu_{E}}(\operatorname{mid}(E)) \quad \text { for any } E \in \mathcal{E}(K), \\
& \left.\left(J_{1} v_{2}\right)\right|_{K}(z)=\left.v_{2}\right|_{K}(z) \\
& \left.\nabla\left(J_{1} v_{2}\right)\right|_{K}(z)= \begin{cases}0 & \text { if } z \in \mathcal{N}(E) \text { for any } z \in \mathcal{N}(K), \\
\left.|\mathcal{T}(K, z)|^{-1} \sum_{T \in \mathcal{T}(K, z)}\left(\nabla v_{1}\right)\right|_{T}(z) & \text { else. }\end{cases}
\end{aligned}
$$

The function $J_{1} v_{2}$ from Definition 6.4 inherits the nodal values as well as the values of the normal derivatives in the edge-midpoints from $v_{2} \in P_{2}(\mathcal{T})$. The values of the derivative e.g. at all interior vertices are computed by averaging over the side-connected $\mathcal{T}(K, z) \subseteq \mathcal{T}(z)$ of cardinality $|\mathcal{T}(K, z)|$. The first alternative in $(6.6),\left.\nabla\left(J_{1} v_{2}\right)\right|_{K}(z)=0$ at all vertices $z \in \mathcal{N}(E)$ of an edge $E \in \mathcal{E}^{\prime}(\partial \Omega):=\mathcal{E}^{\prime} \cap \mathcal{E}(\partial \Omega)$, enforces a vanishing derivative along an edge $E \subset \partial K$ with $\partial v_{2} / \partial \nu_{E}(\operatorname{mid}(E))=0$. The composition $J_{1} v_{2}$ is piecewise $H C T$, but is discontinuous and violates homogeneous boundary conditions in general.

The normalized edge-bubble $b_{E, K}:=30\left(\nu_{K} \cdot \nu_{E}\right) \operatorname{dist}\left(z_{3}, E\right) \varphi_{1}^{2} \varphi_{2}^{2} \varphi_{3} \in P_{5}(\mathcal{T})$ is defined for $K=\operatorname{conv}\left\{z_{1}, z_{2}, z_{3}\right\}=\operatorname{conv}\left\{E, z_{3}\right\}$ with vertex $z_{3}$ opposite to $E$ in $K$. The nodal basis function $\varphi_{j} \equiv \varphi_{z_{j}} \in S_{1}(\mathcal{T})$ is associated with $z_{j}$. The subsequent correction assures that the operator $J_{2}: P_{1}(\mathcal{T}) \rightarrow P_{5}(\mathcal{T})+\prod_{K \in \mathcal{T}} H C T^{\prime}(K)$ preserves the integral means of the normal derivatives $\partial v_{2} / \partial \nu_{E}$ along all edges $E \in \mathcal{E}$.

Definition 6.5 (Local companion $\boldsymbol{J}_{2}$ for piecewise quadratics). For any $K \in \mathcal{T}, v_{2} \in$ $P_{2}(\mathcal{T})$, and $J_{1} v_{2}$ as in Definition 6.4 set

$$
\left.\left(J_{2} v_{2}\right)\right|_{K}:=\left.\left(J_{1} v_{2}\right)\right|_{K}+\sum_{E \in \mathcal{E}(K)}\left(f_{E} \frac{\left.\partial\left(v_{2}-J_{1} v_{2}\right)\right|_{K}}{\partial \nu_{E}} \mathrm{~d} s\right) b_{E, K} \in H C T(K)+P_{5}(K) .
$$


The following properties of the companion operators from Definition 6.4-6.5 will be applied throughout this section.

Lemma 6.2. (a) For any Morley function $v_{M} \in M(\mathcal{T})$ the jumps $\left[J_{1} v_{M}\right]_{E}$ and $\left[\nabla J_{1} v_{M}\right]_{E}$ vanish along any $E \in \mathcal{E}^{\prime}$. In particular, the companion $\left.\left(J_{1} v_{M}\right)\right|_{\omega_{E}} \in H C T^{\prime}(\mathcal{T}(E)):=\{v \in$ $H^{2}\left(\omega_{E}\right):\left.v\right|_{T} \in H C T^{\prime}(T)$ for any $\left.T \in \mathcal{T}(E)\right\}$ is continuously differentiable along any $E \in$ $\mathcal{E}^{\prime} \cap \mathcal{E}(\Omega) ;\left.J_{1} v_{M}\right|_{E}=0$ and $\left.\nabla J_{1} v_{M}\right|_{E}=0$ vanish along any $E \in \mathcal{E}^{\prime}(\partial \Omega)$.

(b) Given any $v_{M} \in M(\mathcal{T})$, the companion $J_{2} v_{M}$ is continuous at the vertices $z \in \mathcal{N}$ and at the midpoints of the edges $E \in \mathcal{E}$; the jumps $\left[J_{2} v_{M}\right]_{E}$ and $\left[\nabla J_{2} v_{M}\right]_{E}$ vanish along $E \in \mathcal{E}^{\prime}$.

(c) If a simplex $K \in \mathcal{T}$ is isolated in the sense that $\mathcal{E}(K) \cap \mathcal{E}^{\prime}=\emptyset$, Definition 6.4-6.5 imply $\left.v_{2}\right|_{K}=\left.\left(J_{1} v_{2}\right)\right|_{K}=\left.\left(J_{2} v_{2}\right)\right|_{K}$ for all $v_{2} \in P_{2}(\mathcal{T})$.

Proof of (a). For any interior edge $E \in \mathcal{E}^{\prime} \cap \mathcal{E}(\Omega)$ with edge-patch $\omega_{E}=\operatorname{int}\left(T_{+} \cup T_{-}\right)$and $\mathcal{T}(E)=\left\{T_{+}, T_{-}\right\}$, Definition 6.1 implies $\mathcal{T}\left(T_{+}, z\right)=\mathcal{T}\left(T_{-}, z\right)$ for any vertex $z \in \mathcal{N}(E)$. Any Morley function $v_{M} \in M(\mathcal{T})$ is continuous at the vertices $z \in \mathcal{N}(E)$ and the normal derivative $\partial v_{M} / \partial \nu_{E}$ is continuous at the edge midpoint $\operatorname{mid}(E)$. Since the coinciding input data at the vertices $z \in \mathcal{N}(E)$ lead to $\left.\nabla_{\mathrm{NC}}\left(J_{1} v_{M}\right)\right|_{T_{+}}(z)=\left.\nabla_{\mathrm{NC}}\left(J_{1} v_{M}\right)\right|_{T_{-}}(z)$ as well, the jumps $\left[J_{1} v_{M}\right]_{E}$ and $\left[\nabla J_{1} v_{M}\right]_{E}$ vanish along any interior edge $E \in \mathcal{E}^{\prime} \cap \mathcal{E}(\Omega)$. The boundary conditions of $v_{M} \in M(\mathcal{T})$ and Definition 6.4 directly imply $\left.J_{1} v_{M}\right|_{E}=0$ and $\left.\nabla J_{1} v_{M}\right|_{E}=0$ along a boundary edge $E \in \mathcal{E}^{\prime}(\partial \Omega)$. This concludes the proof of (a).

Proof of (b). The edge-bubbles $b_{E, K} \in P_{5}(\mathcal{T}) \cap H^{2}(\Omega)$ satisfy $f_{F} \partial b_{E, K} / \partial \nu_{F} \mathrm{~d} s=\delta_{E F}$, $\operatorname{supp}\left(b_{E, K}\right)=K$, and $b_{E, K}(z)$ and $\nabla b_{E, K}(z)$ vanish at any $z \in \mathcal{N}$ for $E, F \in \mathcal{E}$. Hence, $J_{2}$ preserves the integral means of the normal derivatives and (b) follows directly from (a).

Proof of (c). This is elementary for $\mathcal{T}(K, z)=\{K\}$ for all $K \in \mathcal{T}$ with $\mathcal{E}(K) \cap \mathcal{E}^{\prime}=\emptyset$.

The following theorem establishes a local a posteriori approximation error estimate for the operator $J_{2}$ of Definition 6.5 ; recall that $h_{E}=|E|$ is the length of the edge $E \in \mathcal{E}, \mathcal{E}^{\prime}(\partial \Omega):=$ $\mathcal{E}^{\prime} \cap \mathcal{E}(\partial \Omega)$, and $\mathcal{E}(K, z):=\mathcal{E}^{\prime} \cap\left\{E \in \mathcal{E}(z): E \in \partial T_{1} \cap \partial T_{2}\right.$ for $\left.T_{1}, T_{2} \in \mathcal{T}(K, z)\right\}$ in Definition 6.1.

Theorem 6.2. Given (A1), $K \in \mathcal{T}$, and $v_{2} \in P_{2}(\mathcal{T})$, the local companion $J_{2} v_{2}$ of Definition 6.5 satisfies

$$
\begin{aligned}
h_{K}^{-4}\left\|v_{2}-J_{2} v_{2}\right\|_{L^{2}(K)}^{2} & \sum_{z \in \mathcal{N}(K)} \sum_{E \in \mathcal{E}(K, z)} h_{E}^{-1}\left\|\left[\nabla_{\mathrm{NC}} v_{2}\right]_{E}\right\|_{L^{2}(E)}^{2}+\sum_{E \in \mathcal{E}(K) \cap \mathcal{E}^{\prime}(\partial \Omega)} h_{E}^{-1}\left\|\nabla_{\mathrm{NC}} v_{2}\right\|_{L^{2}(E)}^{2} .
\end{aligned}
$$

Proof. Step 1. Definition 6.5 and the triangle inequality show

$$
\left\|v_{2}-J_{2} v_{2}\right\|_{L^{2}(K)} \leq\left\|v_{2}-J_{1} v_{2}\right\|_{L^{2}(K)}+\sum_{E \in \mathcal{E}(K)}\left|f_{E} \partial\left(v_{2}-J_{1} v_{2}\right)\right|_{K} / \partial \nu_{E} \mathrm{~d} s \mid\left\|b_{E, K}\right\|_{L^{2}(K)} .
$$

It holds $\left\|b_{E, K}\right\|_{L^{2}(K)}=2 \sqrt{|K|}^{3} /(|E| \sqrt{2310}) \leq h_{K} \sqrt{|K| / 2310}$ for any $E \in \mathcal{E}(K)$. Lemma D in the appendix quantifies the constant as displayed in the discrete trace inequality

$$
\left|f_{E} \partial\left(v_{2}-J_{1} v_{2}\right)\right|_{K} / \partial \nu_{E} \mathrm{~d} s\left|\leq \sqrt{3 /|K|}\left\|\left.\nabla\left(v_{2}-J_{1} v_{2}\right)\right|_{K} \cdot \nu_{E}\right\|_{L^{2}(K)} .\right.
$$


The combination of the above and the inverse estimate for $H C T^{\prime}(K)$, i.e., piecewise polynomials of degree at most $3[3$, Lemma 4.5 .3$]$ with constant $c_{\mathrm{inv}, 3}$ reveals

$$
\begin{aligned}
\left\|v_{2}-J_{2} v_{2}\right\|_{L^{2}(K)} & \leq\left\|v_{2}-J_{1} v_{2}\right\|_{L^{2}(K)}+3 / \sqrt{770} h_{K}\left\|\nabla\left(v_{2}-J_{1} v_{2}\right)\right\|_{L^{2}(K)} \\
& \leq\left(1+3 c_{\mathrm{inv}, 3} / \sqrt{770}\right)\left\|v_{2}-J_{1} v_{2}\right\|_{L^{2}(K)} .
\end{aligned}
$$

Step 2. For each component $\alpha=1,2$ and any $z \in \mathcal{N}(K)$, let $\psi_{z, \alpha} \in H C T(\mathcal{T})$ denote the nodal basis function with partial derivative $\left(\partial \psi_{z, \alpha} / \partial x_{\alpha}\right)(z)=1$ in direction $x_{\alpha}$, which vanishes for the remaining degrees of freedom. The Hsieh-Clough-Tocher finite element is one in the sense of Ciarlet [16] and so any $\left.v_{2}\right|_{K} \in P_{2}(K) \subset P_{3}(\mathcal{K}(K))$ can be represented by the HCT basis functions. The definition of $J_{1}$ reveals that $\left.\left(v_{2}-J_{1} v_{2}\right)\right|_{K}$ vanishes at the nodes and its normal derivatives vanish at the edge midpoints. Hence this difference belongs to $\operatorname{span}\left\{\psi_{z, \alpha}: z \in \mathcal{N}(K), \alpha=1,2\right\}$. Therefore,

$$
\left\|v_{2}-J_{1} v_{2}\right\|_{L^{2}(K)}=\left\|\sum_{z \in \mathcal{N}(K)} \sum_{\alpha=1,2} \frac{\left.\partial\left(v_{2}-J_{1} v_{2}\right)\right|_{K}}{\partial x_{\alpha}}(z) \psi_{z, \alpha}\right\|_{L^{2}(K)} .
$$

Step 3. The notion of an almost affine family of finite elements in [16, Thm. 6.1.3, p.344] concerns the scaling of the basis functions

$$
\left\|h_{K}^{-2} \psi_{z, \alpha}\right\|_{L^{2}(K)} \lesssim 1 \quad \text { for any } K \in \mathcal{T}, z \in \mathcal{N}(K) \text {, and } \alpha=1,2 \text {. }
$$

The combination with a triangle inequality in (6.7) shows

$$
\left\|h_{K}^{-2}\left(v_{2}-J_{1} v_{2}\right)\right\|_{L^{2}(K)} \lesssim \sum_{z \in \mathcal{N}(K)} \sum_{\alpha=1,2}\left|\frac{\left.\partial\left(v_{2}-J_{1} v_{2}\right)\right|_{K}}{\partial x_{\alpha}}(z)\right|
$$

The non-constructive proof of [16, Thm. 6.1.3] is based on compactness arguments and leaves the constant in (6.8) unquantified.

Step 4. For $v_{2} \in P_{2}(\mathcal{T})$ and $\alpha=1,2$ fixed, the partial derivative $v_{1}:=\partial v_{2} / \partial x_{\alpha} \in P_{1}(\mathcal{T})$ is piecewise affine. To lower a conflict of notation, let $J_{1}^{\prime}$ denote the companion from Definition 6.2 in Section 6.1 and let $J_{1}$ denote the companion from Definition 6.4 above. The nodal values of the derivative $\left.\nabla\left(J_{1} v_{2}\right)\right|_{K}$ in (6.6) coincide component-wise with the nodal values of the companion $J_{1}^{\prime} v_{1}$ in $(6.3)$ applied to $v_{1}$,

$$
\left.\frac{\partial}{\partial x_{\alpha}}\left(J_{1} v_{2}\right)\right|_{K}(z)=\left.\left(J_{1}^{\prime} \frac{\partial v_{2}}{\partial x_{\alpha}}\right)\right|_{K}(z) \quad \text { for any } K \in \mathcal{T} \text { and } z \in \mathcal{N}(K) .
$$

The arguments in Step 3-Step 5 of the proof of Theorem 6.1 apply simultaneously to the components $v_{1}=\partial v_{2} / \partial x_{\alpha} \in P_{1}(\mathcal{T})$ for $\alpha=1,2$ and then lead to

$$
\begin{aligned}
& C_{2}^{-1} \sum_{\alpha=1,2} \sum_{z \in \mathcal{N}(K)}\left|\frac{\left.\partial\left(v_{2}-J_{1} v_{2}\right)\right|_{K}}{\partial x_{\alpha}}(z)\right|^{2} \\
\leq & \sum_{z \in \mathcal{N}(K)} \sum_{E \in \mathcal{E}(K, z)} h_{E}^{-1}\left\|\left[\nabla v_{2}\right]_{E}\right\|_{L^{2}(E)}^{2}+\sum_{E \in \mathcal{E}(K) \cap \mathcal{E}^{\prime}(\partial \Omega)} h_{E}^{-1}\left\|\nabla v_{2}\right\|_{L^{2}(E)}^{2}
\end{aligned}
$$

with $C_{2}=4 \max \left\{2,\left(M_{2}-1\right)\left(2 M_{2}-1\right) /\left(6 M_{2}\right)\right\}$.

Step 5. The combination of Step 1-Step 4 concludes the proof. 

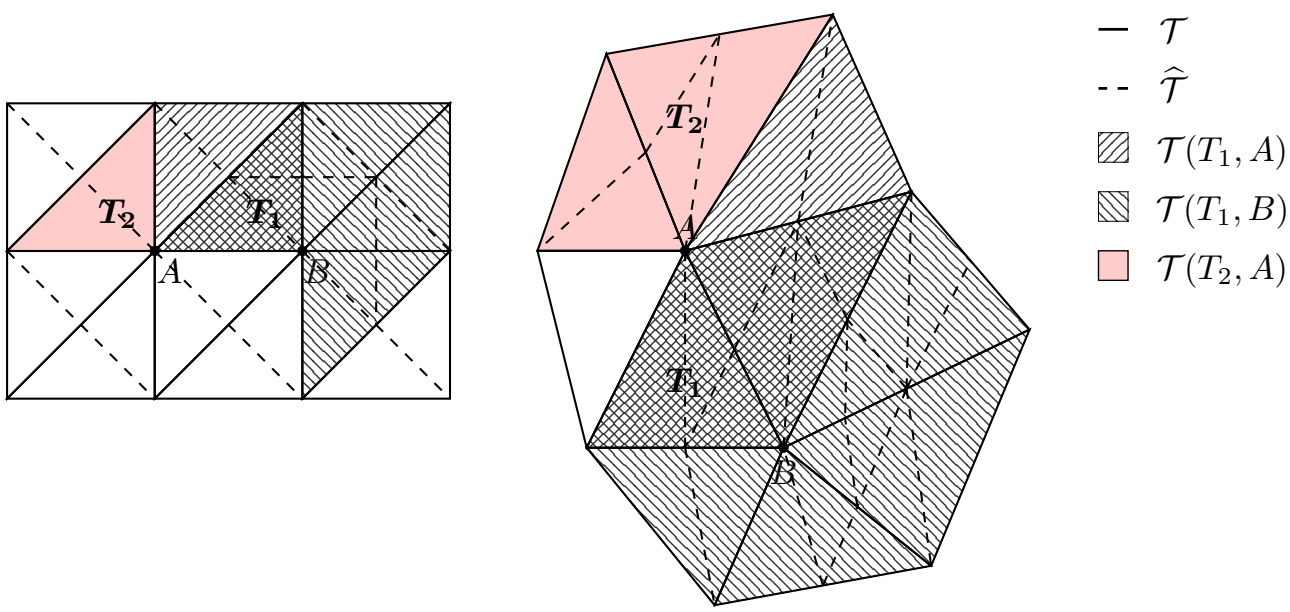

Fig. 6.1. Illustration of the sets $\mathcal{T}(K, z)$ given $\mathcal{F}^{\prime}=\mathcal{F} \backslash \widehat{\mathcal{F}}$ in $(6.10)$.

Remark 6.2. The derivative $D_{N C} v_{M} \in C R_{0}^{1}\left(\mathcal{T} ; \mathbb{R}^{2}\right)$ of a Morley function $v_{M} \in M(\mathcal{T})$ is a Crouzeix-Raviart function in each component. Therefore, the combination of Theorem 6.2 with the Poincaré argument in Remark 6.1 implies, for any $K \in \mathcal{T}$, that

$$
\begin{aligned}
& h_{K}^{-4}\left\|v_{M}-J_{2} v_{M}\right\|_{L^{2}(K)}^{2} \\
\lesssim & \sum_{z \in \mathcal{N}(K)} \sum_{E \in \mathcal{E}(K, z)} h_{E}\left\|\left[D_{N C}^{2} v_{M}\right]_{E} \times \nu_{E}\right\|_{L^{2}(E)}^{2}+\sum_{E \in \mathcal{E}(K) \cap \mathcal{E}^{\prime}(\partial \Omega)} h_{E}\left\|\left[D_{N C}^{2} v_{M}\right]_{E} \times \nu_{E}\right\|_{L^{2}(E)}^{2} .
\end{aligned}
$$

\subsection{Refined Analysis for Crouzeix-Raviart and Morley FEM}

Throughout this section, let $\mathcal{T} \in \mathbb{T}$ be a regular triangulation with set of all sides $\mathcal{F}$ and let $\widehat{\mathcal{T}} \in \mathbb{T}(\mathcal{T})$ be an admissible refinement with set of all sides $\widehat{\mathcal{F}}$. Then define

$$
\mathcal{F}^{\prime}:=\mathcal{F} \backslash \widehat{\mathcal{F}} \subset \mathcal{F}
$$

Fig. 6.1 illustrates the associated sets $\mathcal{T}(K, z)$ from Definition 6.1 for $K \in \mathcal{T}, z \in \mathcal{N}(K)$. The associated set of sides $\mathcal{F}(K, z) \subset \mathcal{F} \backslash \widehat{\mathcal{F}}$ contains only coarse-but-not-fine sides. For a coarse and fine $K \in \mathcal{T} \cap \widehat{\mathcal{T}}$ it holds $\mathcal{T}(K, z)=\{K\}$ as well as $\mathcal{F}(K, z)=\emptyset$ for all $z \in \mathcal{N}(K)$.

The choice of $\mathcal{F}^{\prime}$ in (6.10) allows the definition of an approximation $\widehat{u}_{C R}^{*}$ in (6.11) (resp. $\widehat{u}_{M}^{*}$ in $(6.13)$ below) to the discrete function $u_{C R} \in C R_{0}^{1}(\mathcal{T})$ (resp. $u_{M} \in M(\mathcal{T})$ ). Recall that $\widehat{I}_{\mathrm{NC}}$ from Section 4.1 denotes the nonconforming interpolation operator with respect to $C R(\widehat{\mathcal{T}})$.

Lemma 6.3 $\left(\boldsymbol{u}_{\boldsymbol{C R}}^{*}\right)$. Given any $u_{C R} \in C R_{0}^{1}(\mathcal{T})$ and $(6.10)$ in Definition 6.3,

$$
\widehat{u}_{C R}^{*}:=\widehat{I}_{\mathrm{NC}}\left(J_{n} u_{C R}\right) \in C R_{0}^{1}(\widehat{\mathcal{T}})
$$

is well-defined and satisfies (C4).

Proof. Step 1. Lemma 6.1.b and (6.10) guarantee that $J_{n}\left(u_{C R}\right)$ is continuous in the midpoint of any side $F \in \widehat{\mathcal{F}}$ and vanishes at the midpoint of boundary sides $F \in \widehat{\mathcal{F}}(\partial \Omega)$. Hence the nonconforming interpolation $\widehat{u}_{C R}^{*}=\widehat{I}_{\mathrm{NC}}\left(J_{n} u_{C R}\right) \in C R_{0}^{1}(\widehat{\mathcal{T}})$ is well defined and admits homogeneous boundary conditions. 
Step 2. The correction with the side-bubble functions in Definition 6.3 leads to the identity

$$
f_{F} \widehat{u}_{C R}^{*} \mathrm{~d} s=f_{F} u_{C R} \mathrm{~d} s \quad \text { for all } F \in \mathcal{F} .
$$

The integral means are traces on the neighbouring simplices $T_{ \pm}$on $F$ and those values are independent of $T_{+}$or $T_{-}$for an interior side. This and the definition of $I_{N C}$ imply (C4).

Recall that $\widehat{I}_{M}$ from Section 5.1 denotes the interpolation operator with respect to $\mathcal{M}(\widehat{\mathcal{T}})$.

Lemma 6.4 $\left(\boldsymbol{u}_{M}^{*}\right)$. Given any $u_{M} \in M(\mathcal{T})$ and (6.10) in Definition 6.5,

$$
\widehat{u}_{M}^{*}:=\widehat{I}_{\mathrm{M}}\left(J_{2} u_{M}\right) \in M(\widehat{\mathcal{T}})
$$

is well-defined and satisfies (C4).

Proof. Step 1. Lemma 6.2.b and (6.10) guarantee the continuity of $\nabla J_{2}\left(u_{M}\right)$ at the midpoints of $E \in \widehat{\mathcal{E}}$ and of $J_{2}\left(u_{M}\right)$ at the new vertices $\widehat{\mathcal{N}}$ (either $z \in \mathcal{N} \cap \widehat{\mathcal{N}}$ or $z \in E \in \mathcal{E}^{\prime}$ ). In particular, $J_{2}\left(u_{M}\right)$ vanishes at all vertices in $\widehat{\mathcal{N}}(\partial \Omega)$ and $\nabla J_{2}\left(u_{M}\right)$ vanishes at the midpoints of all edges in $\widehat{\mathcal{E}}(\partial \Omega)$. Hence the Morley interpolation $\widehat{u}_{M}^{*}=\widehat{I}_{\mathrm{M}}\left(J_{2} u_{M}\right) \in M^{\prime}(\widehat{\mathcal{T}})$ is well defined and admits homogeneous boundary conditions.

Step 2. The definition of $\widehat{I}_{M}$ and Definition 6.5 show that the nodal values $\widehat{u}_{M}^{*}(z)=u_{M}(z)$ coincide for all $z \in \mathcal{N}$ and the correction with the edge-bubble functions guarantees

$$
f_{E} \frac{\partial \widehat{u}_{M}^{*}}{\partial \nu_{E}} \mathrm{~d} s=f_{E} \frac{\partial u_{M}}{\partial \nu_{E}} \mathrm{~d} s \quad \text { for all } E \in \mathcal{E}
$$

The integral means are traces on the neighbouring simplices $T_{ \pm}$on $E$ and those values are independent of $T_{+}$or $T_{-}$for an interior edge. This and the definition of $I_{M}$ imply (C4).

The estimate (6.1) follows by collecting the above results in Theorem 6.3 resp. 6.4 below.

Theorem 6.3. Given $u_{C R} \in C R_{0}^{1}(\mathcal{T})$ and its approximation $\widehat{u}_{C R}^{*} \in C R_{0}^{1}(\widehat{\mathcal{T}})$ in $(6.11)$,

$$
\left\|\widehat{u}_{C R}^{*}-u_{C R}\right\|_{h}^{2} \leq c_{\mathrm{inv}, n}^{2} C_{n}^{\prime} M_{2} \sum_{F \in \mathcal{F} \backslash \widehat{\mathcal{F}}} h_{F}\left\|\left[\nabla_{\mathrm{NC}} u_{C R}\right]_{F} \times \nu_{F}\right\|_{L^{2}(F)}^{2}
$$

holds with $c_{\mathrm{inv}, n}$ from the inverse estimate for piecewise polynomials up to degree $n, C_{n}^{\prime}$ from Remark 6.1, and $M_{2}$ from assumption (A1).

Proof. Conditions (C3)-(C5) for $\widehat{I}_{\mathrm{NC}}$ resp. $\widehat{u}_{C R}^{*}$ lead to (3.3) and an inverse estimate with constant $c_{\mathrm{inv}, n}$ for polynomial functions of degree at most $n$ leads to

$$
\left\|\widehat{u}_{C R}^{*}-u_{C R}\right\|_{h}=\left\|D_{\mathrm{NC}}\left(\widehat{u}_{C R}^{*}-u_{C R}\right)\right\|_{L^{2}(\mathcal{T} \backslash \widehat{\mathcal{T}})} \leq c_{\mathrm{inv}, n}\left\|h_{\mathcal{T}}^{-1}\left(J_{n} u_{C R}-u_{C R}\right)\right\|_{L^{2}(\mathcal{T} \backslash \widehat{\mathcal{T}})} .
$$

Theorem 6.1 and Remark 6.1 conclude the proof of $(6.1)$ for $\mathcal{F}(K, z) \subset \mathcal{F} \backslash \widehat{\mathcal{F}}$ for any $K \in \mathcal{T}$ and $z \in \mathcal{N}(K)$.

Corollary 6.1. The discrete reliability (dRel) holds for the Crouzeix-Raviart FEM with $\mathcal{T} \backslash \widehat{\mathcal{T}}$ replacing $\mathcal{R}$ and the constant $\Lambda_{\text {drel }}:=(1+1 / \sqrt{2}) \max \left\{\sqrt{19 / 48}, c_{\mathrm{inv}, n} \sqrt{C_{n}^{\prime} M_{2}}\right\}$. 
Proof. Theorem 6.3 shows in particular (1.4) with $\mathcal{T} \backslash \widehat{\mathcal{T}}$ replacing $\mathcal{R}$ and constant $\Lambda_{2}^{2}=$ $c_{\text {inv }, n}^{2} C_{n}^{\prime} M_{2}$. Section 4 proves $(\mathrm{C} 1)-(\mathrm{C} 3)$ for $I_{\mathrm{NC}}$ and Lemma 6.3 proves $(\mathrm{C} 4)$. Hence Theorem 3.1 implies (1.3). The combination of (1.3)-(1.4) concludes the proof of (dRel).

Corollary 6.2 (Constants in 2D). The constant $\Lambda_{\text {drel }}$ in (dRel) is bounded in terms of the minimal angle $\omega_{0}$ and $M_{2}=\sup _{z \in \mathcal{N}, \mathcal{T} \in \mathbb{T}}|\mathcal{T}(z)| \leq 2 \pi / \omega_{0}$ by

$$
\begin{aligned}
& \Lambda_{\text {drel }}:=\frac{1+1 / \sqrt{2}}{12} \max \left\{\sqrt{57}, C_{J} \sqrt{\max \left\{12 M_{2},\left(M_{2}-1\right)\left(2 M_{2}-1\right)\right\}}\right\}, \\
& C_{J}^{2}:=\frac{97}{4} \cot \left(\omega_{0}\right)\left(2 \cot \left(\omega_{0}\right)-\cot \left(2 \omega_{0}\right)\right)+24 \cot \left(\omega_{0}\right) \sqrt{\left(2 \cot \left(\omega_{0}\right)-\cot \left(2 \omega_{0}\right)\right)^{2}-3} .
\end{aligned}
$$

Proof. Given $\Lambda_{1}=\sqrt{19 / 48}$ from Section 4.2 in (1.3), it remains to compute the constant $\Lambda_{2}$ in (1.4). Corollary 6.1 proves $\Lambda_{2}=c_{i n v, 2} \sqrt{C_{2}^{\prime} M_{2}}$. The following calculation circumvent the computation of the constant $c_{i n v, 2}$ in the inverse estimate for piecewise quadratics. From the conditions $(\mathrm{C} 3)-(\mathrm{C} 5)$ for $\widehat{I}_{\mathrm{NC}}$ resp. $\widehat{u}_{C R}^{*}$ follows $(3.3)$. For each $K \in \mathcal{T} \backslash \widehat{\mathcal{T}}$ the triangle inequality leads to

$$
\begin{aligned}
& \left\|\nabla\left(u_{C R}-J_{2} u_{C R}\right)\right\|_{L^{2}(K)} \\
\leq & \left\|\nabla\left(u_{C R}-J_{1} u_{C R}\right)\right\|_{L^{2}(K)}+\sum_{F \in \mathcal{F}(K)}\left|f_{F}\left(u_{C R}-J_{1} u_{C R}\right)\right|_{K} \mathrm{~d} s \mid\left\|\nabla b_{F}\right\|_{L^{2}(K)} .
\end{aligned}
$$

If $\alpha, \beta, \gamma$ denote the interior angles in $K,\left\|\nabla b_{F}\right\|_{L^{2}(K)}=\sqrt{\cot \alpha+\cot \beta+\cot \gamma} /(12 \sqrt{3})$. A maximisation shows

$$
\left\|\nabla b_{F}\right\|_{L^{2}(K)} \leq \sqrt{2 \cot \left(\omega_{0}\right)-\cot \left(2 \omega_{0}\right)} /(12 \sqrt{3})
$$

The combination with Lemma $\mathrm{D}$ in the appendix for any $F \in \mathcal{F}(K)$ and $h_{K}^{2} \leq 4|K| \cot \left(\omega_{0}\right)$ implies

$$
\begin{aligned}
& \sum_{F \in \mathcal{F}(K)}\left|f_{F}\left(u_{C R}-J_{1} u_{C R}\right)\right|_{K} \mathrm{~d} s \mid\left\|\nabla b_{F}\right\|_{L^{2}(K)} \\
\leq & \frac{\sqrt{\cot \left(\omega_{0}\right)\left(2 \cot \left(\omega_{0}\right)-\cot \left(2 \omega_{0}\right)\right)}}{2 h_{K}}\left\|u_{C R}-J_{1} u_{C R}\right\|_{L^{2}(K)} .
\end{aligned}
$$

On the other hand, [10, Lem. 4.10] establishes the constant $c_{\mathrm{inv}, 1}^{2}=24 \cot \left(\omega_{0}\right)\left(2 \cot \left(\omega_{0}\right)-\right.$ $\left.\cot \left(2 \omega_{0}\right)+\sqrt{\left(\cot \left(\omega_{0}\right)-\cot \left(2 \omega_{0}\right)\right)^{2}-3}\right)$ in the inverse estimate for affine functions. Therefore, $\left\|\nabla\left(u_{C R}-J_{2} u_{C R}\right)\right\|_{L^{2}(K)} \leq C_{J} h_{K}^{-1}\left\|u_{C R}-J_{1} u_{C R}\right\|_{L^{2}(K)}$ holds with the constant $C_{J}$. The combination of Step 2-Step 4 in the proof of Theorem 6.1 and Remark 6.1 shows that $C_{2}:=$ $\max \left\{2,\left(M_{2}-1\right)\left(2 M_{2}-1\right) /\left(6 M_{2}\right)\right\} C_{J}^{2} / 24$ satisfies

$$
\begin{aligned}
& C_{2}^{-1}\left\|\nabla\left(u_{C R}-J_{2} u_{C R}\right)\right\|_{L^{2}(K)}^{2} \\
\leq & \sum_{F \in \mathcal{F}(K) \cap \mathcal{F}^{\prime}(\partial \Omega)} h_{F}\left\|\left[\nabla_{N C} u_{C R}\right]_{F} \times \nu_{F}\right\|_{L^{2}(F)}^{2}+\sum_{z \in \mathcal{N}(K)} \sum_{F \in \mathcal{F}(K, z)} h_{F}\left\|\left[\nabla_{N C} u_{C R}\right]_{F} \times \nu_{F}\right\|_{L^{2}(F)}^{2} .
\end{aligned}
$$

The sum over all $K \in \mathcal{T} \backslash \widehat{\mathcal{T}}$ and an overlap argument for $\mathcal{F}(K, z) \subset \mathcal{F} \backslash \widehat{\mathcal{F}}$ conclude the proof of (1.4) with $\lambda_{2}^{2}:=M_{2} C_{2}$. The combination of (1.3)-(1.4) proves (dRel). 
Example 6.2. Given a triangulation with a minimal angle $\omega_{0}=45^{\circ}$ and $M_{2} \leq 8$, for instance, in a triangulation in right isosceles triangles, $C_{J}=\sqrt{145 / 2} \leq 8.5147$ and $\Lambda_{\text {drel }}=$ $(1+1 / \sqrt{2}) \sqrt{5075 / 96} \leq 12.4121$ follows, a significant improvement over [10, Ex. 6.3].

Theorem 6.4. Given (A1), $u_{M} \in M(\mathcal{T})$, and its approximation $\widehat{u}_{M}^{*} \in M(\widehat{\mathcal{T}})$ in (6.13),

$$
\left\|\widehat{u}_{M}^{*}-u_{M}\right\|_{h}^{2} \lesssim \sum_{E \in \mathcal{E} \backslash \widehat{\mathcal{E}}} h_{E}\left\|\left[D_{\mathrm{NC}}^{2} u_{M}\right]_{E} \times \nu_{E}\right\|_{L^{2}(E)}^{2}
$$

Proof. Conditions (C3)-(C5) for $\widehat{I}_{M}$ resp. $\widehat{u}_{M}^{*}$ lead to (3.3) and an inverse estimate for piecewise polynomial functions shows

$$
\left\|\widehat{u}_{M}^{*}-u_{M}\right\|_{h}=\left\|D_{\mathrm{NC}}^{2}\left(\widehat{u}_{M}^{*}-u_{M}\right)\right\|_{L^{2}(\mathcal{T} \backslash \widehat{\mathcal{T}})} \lesssim\left\|h_{\mathcal{T}}^{-2}\left(J_{2} u_{M}-u_{M}\right)\right\|_{L^{2}(\mathcal{T} \backslash \widehat{\mathcal{T}})} .
$$

Theorem 6.2 and Remark 6.2 conclude the proof of $(6.1)$ for $\mathcal{E}(K, z) \subset \mathcal{E} \backslash \widehat{\mathcal{E}}$ for any $K \in \mathcal{T}$ and $z \in \mathcal{N}(K)$.

Corollary 6.3. The discrete reliability (dRel) holds for the Morley FEM with $\mathcal{T} \backslash \widehat{\mathcal{T}}$ replacing $\mathcal{R}$.

Proof. Theorem 6.4 shows in particular (1.4) with $\mathcal{T} \backslash \widehat{\mathcal{T}}$ replacing $\mathcal{R}$. Section 5 proves $(\mathrm{C} 1)-$ (C3) for $I_{M}$ and Lemma 6.4 proves (C4). Hence Theorem 3.1 implies (1.3). The combination of (1.3)-(1.4) concludes the proof of (dRel).

Acknowledgments. This work has been supported by the Deutsche Forschungsgemeinschaft (DFG) in the Priority Program 1748 Reliable simulation techniques in solid mechanics. Development of non-standard discretization methods, mechanical and mathematical analysis under the project CA 151/22. The second author is supported by the Berlin Mathematical School.

\section{Appendix}

The appendix presents some optimal constants in fundamental inequalities.

\section{Appendix A}

The subsequent inverse estimate displays an optimal constant $(k+1) / \sqrt{b-a}$.

Lemma A. Any polynomial $f$ of degree at most $k \in \mathbb{N}$ in a non-void bounded open interval $(a, b)$ satisfies

$$
|f(a)| \leq \frac{k+1}{\sqrt{b-a}}\|f\|_{L^{2}(a, b)} .
$$

For any constant $C<(k+1) / \sqrt{b-a}$, there exists some polynomial $f$ of degree at most $k$ with $C\|f\|_{L^{2}(a, b)}<|f(a)|$.

Proof. An affine transformation of the interval $(a, b)$ onto $(-1,+1)$ shows that, without loss of generality, one may consider the particular case $a=-1$ and $b=1$. The Legendre polynomials $p_{m} \in P_{k}[-1,+1]$ are defined in many ways. For instance via the initialization $p_{0}=1$ and $p_{1}(x)=x$ followed by the recursion formula

$$
(m+1) p_{m+1}(x)=(2 m+1) x p_{m}(x)-m p_{m-1}(x) \quad \text { for } m=1,2,3, \ldots
$$


Then the Legendre polynomials $p_{m}$ are pairwise orthogonal with

$$
\int_{-1}^{+1} p_{m}(x) p_{n}(x) \mathrm{d} x=\frac{2 \delta_{m n}}{2 m+1}
$$

and normalized by $p_{m}(-1)=(-1)^{m} p_{m}(1)=(-1)^{m}$ for $m, n \in \mathbb{N}_{0}$. The polynomial $f(x)=$ $\sum_{j=0}^{k} a_{j} p_{j}$ for some coefficients $a_{0}, a_{1}, \ldots, a_{k} \in \mathbb{R}$ satisfies

$$
\|f\|_{L^{2}(-1,1)}^{2}=\sum_{j=0}^{k} \frac{2 a_{j}^{2}}{2 j+1} \quad \text { and } \quad f(-1)=\sum_{j=0}^{k}(-1)^{j} a_{j} .
$$

The latter value is the scalar product in $\mathbb{R}^{k+1}$ of the vectors $\left((-1)^{j} a_{j} \sqrt{2 /(2 j+1)}: j=\right.$ $0,1, \ldots, k)$ and $(\sqrt{(2 j+1) / 2}: j=0,1, \ldots, k)$. The Cauchy inequality in $\mathbb{R}^{k+1}$ shows

$$
|f(-1)| \leq\|f\|_{L^{2}(-1,1)} \sqrt{\sum_{j=0}^{k}(j+1 / 2)}=\frac{k+1}{\sqrt{2}}\|f\|_{L^{2}(-1,1)} .
$$

Notice that the Cauchy inequality is an equality for certain coefficients and so the assertion is sharp in the sense stated in the second half of the lemma.

\section{Appendix B}

This section utilizes some language of graph theory and concerns an undirected graph $G$ as a pair $(\{1, \ldots, n\}, \mathcal{E})$ of a set of vertices $\{1, \ldots, n\}$ (fixed with $n$ in this section and so neglected in the notation) and a set $\mathcal{E}$ of edges $\{j, k\}$ with $j, k \in\{1, \ldots, n\}$ and $j \neq k$. The graph $G$ (identified with $\mathcal{E}$ ) is connected if for all $j, k \in\{1, \ldots, n\}$ and $j \neq k$ there are $m \in$ $\{1,2,3, \ldots\}$ edges $\left\{\alpha_{1}, \alpha_{2}\right\}, \ldots,\left\{\alpha_{m}, \alpha_{m+1}\right\} \in \mathcal{E}$ with $\alpha_{1}=j$ and $\alpha_{m+1}=k$. The set of all connected graphs $G$ over the set $\{1, \ldots, n\}$ is identified with the set $\mathcal{C}(n)$ of all sets of edges $\mathcal{E}$; so $(\{1, \ldots, n\}, \mathcal{E})$ is connected is abbreviated as $\mathcal{E} \in \mathcal{C}(n)$.

Given $x_{1}, \ldots, x_{n} \in \mathbb{R}$ the goal is to minimize

$$
f(\mathcal{E}):=\sum_{\{j, k\} \in \mathcal{E}}\left(x_{j}-x_{k}\right)^{2} \text { over all } \mathcal{E} \in \mathcal{C}(n) .
$$

Since permutations $\sigma$ of the set $\{1, \ldots, n\}$ transform $\mathcal{E} \in \mathcal{C}(n)$ into $\sigma(\mathcal{E}):=\{\{\sigma(j), \sigma(k)\}$ : $\{j, k\} \in \mathcal{E}\} \in \mathcal{C}(n)$, without loss of generality, we may and will assume that the enumeration orders the real values $x_{1} \leq x_{2} \leq \cdots \leq x_{n}$.

Lemma B. Any vector $x=\left(x_{1}, \ldots, x_{n}\right) \in \mathbb{R}^{n}$ with $n \in \mathbb{N}$ and $x_{1} \leq x_{2} \leq \cdots \leq x_{n}$ and the function $f(\mathcal{E}):=\sum_{\{j, k\} \in \mathcal{E}}\left(x_{j}-x_{k}\right)^{2}$ satisfy

$$
m(x):=\min _{\mathcal{E} \in \mathcal{C}(n)} f(\mathcal{E})=\sum_{j=1}^{n-1}\left(x_{j+1}-x_{j}\right)^{2} .
$$

Proof. (1) For any $n \in \mathbb{N}$ and $x \in \mathbb{R}^{n}$ the minimum $m(x):=\min _{\mathcal{E} \in \mathcal{C}(n)} f(\mathcal{E})$ (where below $f$ applies to any vector of any length) is attained for some $\mathcal{E} \in \mathcal{C}(n)$ with $|\mathcal{E}|=n-1$.

It is known in graph theory that loops can be avoided by certain cuts and any cut means a reduction of the target functional. Therefore, we may and will assume without loss of generality, 
that $\mathcal{E} \in \mathcal{C}(n)$ is a tree. There is only a finite number of trees for a fixed number of vertices $n$ and so the minimum $m(x)$ is attained for at least one of them.

(2) Given any $x_{1} \leq x_{2} \leq \cdots \leq x_{n}$ it holds

$$
m(x):=\min _{\mathcal{E} \in \mathcal{C}(n)} \sum_{\{j, k\} \in \mathcal{E}}\left(x_{j}-x_{k}\right)^{2}=\sum_{j=1}^{n-1}\left(x_{j+1}-x_{j}\right)^{2} .
$$

The proof is by mathematical induction. The assertion holds for $n=1$ (pathological) and $n=2$ (trivial), so suppose it holds for some $n \geq 2$ and all $x_{1} \leq x_{2} \leq \cdots \leq x_{n}$. Given $\widehat{x}=\left(x_{1}, \ldots, x_{n+1}\right) \in \mathbb{R}^{n+1}$ with $x_{1} \leq \cdots \leq x_{n} \leq x_{n+1}$, let $\widehat{\mathcal{E}} \in \mathcal{C}(n+1)$ be a minimizer with $f(\widehat{\mathcal{E}})=m(\widehat{x})$.

Step 1. Since $\widehat{\mathcal{E}} \in \mathcal{C}(n+1)$ is connected, there is a path $\left\{\alpha_{1}, \alpha_{2}\right\}, \ldots,\left\{\alpha_{m}, \alpha_{m+1}\right\} \in \widehat{\mathcal{E}}$ of length $m \in \mathbb{N}$ with $\alpha_{1}=n+1$ and $\alpha_{m+1}=n$. The numbers $\alpha_{1}, \ldots, \alpha_{m+1}$ can be chosen pairwise distinct (as loops may be excluded). Then $\mathcal{E}^{\prime}:=\widehat{\mathcal{E}} \backslash\left\{\left\{n+1, \alpha_{2}\right\}\right\}$ and $\widetilde{\mathcal{E}}:=\mathcal{E}^{\prime} \cup\{\{n, n+1\}\}$ lead to $\widetilde{\mathcal{E}} \in \mathcal{C}(n+1)$ and

$$
f(\widehat{\mathcal{E}})-f(\widetilde{\mathcal{E}})=\left(x_{n+1}-x_{\alpha_{2}}\right)^{2}-\left(x_{n}-x_{n+1}\right)^{2} \geq 0\left(\text { since } x_{\alpha_{2}} \leq x_{n} \leq x_{n+1}\right) .
$$

Consequently, there exists a minimizer $\widehat{\mathcal{E}} \in \mathcal{C}(n+1)$ with $\{n, n+1\} \in \widehat{\mathcal{E}}$.

Step 2. For any $k \in\{1,2, \ldots, n-1\}$ with $\{k, n+1\} \in \widehat{\mathcal{E}}$ consider $\mathcal{E}^{\prime}:=\widehat{\mathcal{E}} \backslash\{\{k, n+1\}\}$ and $\widetilde{\mathcal{E}}:=\mathcal{E}^{\prime} \cup\{\{k, n\}\}$. Then $\widetilde{\mathcal{E}} \in \mathcal{C}(n+1)$ is connected (for $\{n, n+1\} \in \widehat{\mathcal{E}} \in \mathcal{C}(n+1)$ ). Moreover,

$$
f(\widehat{\mathcal{E}})-f(\widetilde{\mathcal{E}})=\left(x_{n+1}-x_{k}\right)^{2}-\left(x_{n}-x_{k}\right)^{2} \geq 0 \quad\left(\text { since } x_{k} \leq x_{n} \leq x_{n+1}\right) .
$$

Step 3. A finite number of changes as in Step 2 leads to a minimizer $\widehat{\mathcal{E}} \in \mathcal{C}(n+1)$ of $f$ with $\{n, n+1\} \in \widehat{\mathcal{E}}$ and $\{k, n+1\} \notin \widehat{\mathcal{E}}$ for all $k=1, \ldots, n-1$.

Step 4. Given a minimizer $\widehat{\mathcal{E}} \in \mathcal{C}(n+1)$ from Step 3, the set $\mathcal{E}^{\prime}:=\widehat{\mathcal{E}} \backslash\{n+1, n\} \in$ $\mathcal{C}(n)$ is connected and the induction hypothesis guarantees for $x:=\left(x_{1}, \ldots, x_{n}\right)$ that $m(x)=$ $\sum_{j=1}^{n-1}\left(x_{j+1}-x_{j}\right)^{2} \leq f\left(\mathcal{E}^{\prime}\right)$ (where the abbreviation $f$ applies to $\mathcal{E}^{\prime}$ as well). Consequently,

$$
m(\widehat{x})=f(\widehat{\mathcal{E}})=f\left(\mathcal{E}^{\prime}\right)+\left(x_{n+1}-x_{n}\right)^{2} \geq \sum_{j=1}^{n}\left(x_{j+1}-x_{j}\right)^{2} .
$$

Since $\{\{1,2\}, \ldots,\{n, n+1\}\} \in \mathcal{C}(n+1)$ is in the competition with

$$
f(\{\{1,2\},\{2,3\}, \ldots,\{n, n+1\}\})=\sum_{j=1}^{n}\left(x_{j+1}-x_{j}\right)^{2} \leq m(\widehat{x})=f(\widehat{\mathcal{E}})
$$

and $\widehat{\mathcal{E}}$ is a minimizer, the claim $m(\widehat{x})=\sum_{j=1}^{n}\left(x_{j+1}-x_{j}\right)^{2}$ follows.

\section{Appendix C}

The subsequent estimate holds with the optimal constant $(n-1)(2 n-1) /(6 n)$.

Lemma C. Any $x \in \mathbb{R}^{n}, n \in \mathbb{N}$, with vanishing sum $x \cdot(1, \ldots, 1)=\sum_{j=1}^{n} x_{j}=0$ satisfies

$$
\max _{j=1}^{n}\left|x_{j}\right|^{2} \leq \frac{(n-1)(2 n-1)}{6 n} \sum_{j=1}^{n-1}\left(x_{j+1}-x_{j}\right)^{2} .
$$

For any constant $C<(n-1)(2 n-1) /(6 n)$, there exists some $x \in \mathbb{R}^{n}$ with $x \cdot(1, \ldots, 1)=0$ and $C \sum_{j=1}^{n-1}\left(x_{j+1}-x_{j}\right)^{2}<\max _{j=1}^{n}\left|x_{j}\right|^{2}$. 
Proof. The assertion holds for $n=1$ (pathological) and $n=2$ (trivial). A scaling argument for $n \geq 3$ proves that the multiplicative constant in the asserted inequality is the reciprocal of

$$
\mu(n):=\min _{x \in \mathcal{A}(n)} f(x) \text { for } f(x):=\sum_{j=1}^{n-1}\left(x_{j+1}-x_{j}\right)^{2}
$$

and $\mathcal{A}(n):=\left\{x \in \mathbb{R}^{n}: x \perp(1, \ldots, 1)\right.$ and $\left.\|x\|_{\infty}=1\right\}$. The arguments of Lemma B (with a change of all signs if necessary) lead to the identity

$$
\mu(n)=\min _{x \in \mathcal{B}(n)} f(x) \text { for } \mathcal{B}(n)=\left\{x \in \mathbb{R}^{n}: x \perp(1, \ldots, 1) \text { and }-1 \leq x_{1} \leq \cdots \leq x_{n}=1\right\} .
$$

Any $x \in \mathcal{B}(n)$ is transformed into $y=\left(y_{1}, \ldots, y_{n-1}\right) \in \mathbb{R}^{n-1}$ (recall $n \geq 3$ ) via

$$
y_{j}:=x_{j+1}-x_{j} \text { for all } j=1, \ldots, n-1, \text { so that } f(x)=|y|^{2}:=\sum_{j=1}^{n-1} y_{j}^{2} \text { and } y \geq 0
$$

(with $y \geq 0$ understood componentwise as $y_{j} \geq 0$ for $j=1, \ldots, n-1$ ). Since

$$
x_{k}=1-\sum_{j=k}^{n-1} y_{j} \text { for all } k=1, \ldots, n
$$

(the empty sum is zero), the condition $x \perp(1, \ldots, 1)$ is equivalent to $n=(1, \ldots, n-1) \cdot y$ with the scalar product $\cdot$ in $\mathbb{R}^{n-1}$. The restriction $-1 \leq x_{1} \leq \cdots \leq x_{n}=1$ is equivalent to $y \geq 0$ and $(1, \ldots, 1) \cdot y \leq 2$.

In conclusion, for $x \in \mathbb{R}^{n}$ and $y \in \mathbb{R}^{n-1}$ with $(\mathrm{C} 1), x \in \mathcal{B}(n)$ is equivalent to

$$
y \in \mathcal{C}(n):=\left\{0 \leq y \in \mathbb{R}^{n-1}:(1, \ldots, n-1) \cdot y=n \text { and }(1, \ldots, 1) \cdot y \leq 2\right\} .
$$

To determine $\min _{x \in \mathcal{B}(n)} f(x)=\min _{y \in \mathcal{C}(n)}|y|^{2}$, suppose that $y \in \mathcal{C}(n)$ and utilize a Cauchy inequality for

$$
n=(1,2, \ldots, n-1) \cdot y \leq|y| \sqrt{\sum_{j=1}^{n-1} j^{2}}=|y| \sqrt{\frac{(n-1) n(2 n-1)}{6}} .
$$

Consequently, $\frac{6 n}{(n-1)(2 n-1)} \leq|y|^{2}$. Since $y \in \mathcal{C}(n)$ is arbitrary, this proves one inequality in the claim

$$
\mu(n)=\frac{6 n}{(n-1)(2 n-1)} \text { for } n \in \mathbb{N} .
$$

To prove the reverse inequality, let $\lambda:=n\left(\sum_{j=1}^{n-1} j^{2}\right)^{-1}=\frac{6}{(n-1)(2 n-1)}>0$ and $y=\lambda(1,2, \ldots, n-$ 1) $\geq 0$ with $y \cdot(1,2, \ldots, n-1)=n$ and

$$
(1, \ldots, 1) \cdot y=\lambda \sum_{j=1}^{n-1} j=\frac{\lambda(n-1) n}{2}=\frac{3 n}{2 n-1} \leq 2 \quad \text { for } n \geq 2 .
$$

Consequently, $y \in \mathcal{C}(n)$ and $\mu(n) \leq|y|^{2}=\frac{6 n}{(n-1)(2 n-1)}$. This concludes the proof of (C3). It also proves the asserted optimality of the displayed constant. 


\section{Appendix D}

This section is devoted to some discrete trace inequality for affine functions. The first estimate in (D1) is an equality for the constant function $f \equiv 1$ in any space dimension. The affine function $f$ with $\left.f\right|_{F_{j}} \equiv 1$ on $F_{j} \in \mathcal{F}(K)$ and $f\left(P_{j}\right)=-n / 2$ at the vertex $P_{j} \in \mathcal{N}(K)$ opposite to $F_{j}$ leads to an equality in the second estimate in (D1). The third estimate in (D1) is an equality for the affine function with $\left.f\right|_{F_{j}}=1$ and $f\left(P_{j}\right)=-(n+1)$.

Lemma D. Let $K \subset \mathbb{R}^{n}$ be a simplex of positive volume $|K|$ with the set $\mathcal{F}(K)=\left\{F_{0}, F_{1}, \ldots\right.$, $\left.F_{n}\right\}$ of its sides and the set $\mathcal{N}(K)=\left\{P_{0}, P_{1}, \ldots, P_{n}\right\}$ of its vertices. Then any $f \in P_{1}(K)$ satisfies

$$
\left\{\sum_{k=0}^{n}\left|f_{F_{k}} f \mathrm{~d} s\right|^{2}, \frac{n}{2} \max _{j=0, \ldots, n}\left|f_{F_{j}} f \mathrm{~d} s\right|^{2}, \max _{j=0, \ldots, n} \frac{\left|f\left(P_{j}\right)\right|^{2}}{(n+1)}\right\} \leq \frac{n+1}{|K|}\|f\|_{L^{2}(K)}^{2} .
$$

Proof. Let $P_{k}$ denote the vertex opposite to the side $F_{k}$ in $K=\operatorname{conv}\left\{P_{k}, F_{k}\right\}$ and set $x_{k}:=f\left(P_{k}\right)$ for $k=0, \ldots n$. For an affine $f \in P_{1}(K)$ and a side $F_{k} \in \mathcal{F}(K)$ the integral mean $f_{F_{k}} f \mathrm{~d} s=f\left(\operatorname{mid}\left(F_{k}\right)\right)=\sum_{\substack{j=0 \\ j \neq k}}^{n} x_{j} / n$ is rewritten with $s:=\sum_{k=0}^{n} x_{k}$ and $|x|^{2}:=\sum_{k=0}^{n} x_{k}^{2}$,

$$
n^{2} \sum_{k=0}^{n}\left|f_{F_{k}} f \mathrm{~d} s\right|^{2}=\sum_{k=0}^{n}\left(\sum_{\substack{j=0 \\ j \neq k}}^{n} x_{j}\right)^{2}=\sum_{k=0}^{n}\left(s-x_{k}\right)^{2}=|x|^{2}+(n-1) s^{2} .
$$

The Cauchy-Schwarz inequality implies $s^{2} \leq(n+1)|x|^{2}$ and so $(n-2) s^{2} \leq\left(n^{2}-n-2\right)|x|^{2}$, which is equivalent to

$$
|x|^{2}+(n-1) s^{2} \leq \frac{n^{2}}{n+2}\left(|x|^{2}+s^{2}\right) .
$$

The combination with the local mass matrix for the $P_{1}$-conforming FEM in (6.4), namely

$$
\|f\|_{L^{2}(K)}^{2}=\frac{|K|}{(n+1)(n+2)}\left(|x|^{2}+s^{2}\right),
$$

concludes the proof of the first inequality in (D1).

Without loss of generality assume $j=0$ in the remaining estimates in (D1). Let $x:=$ $\left(x_{0}, x_{1}, \ldots, x_{n}\right) \in \mathbb{R}^{n+1}$, to deduce

$$
n^{2}\left|f_{F_{0}} f \mathrm{~d} s\right|^{2}=\left(\sum_{j=1}^{n} x_{j}\right)^{2}=|x \cdot(0,1, \ldots, 1)|^{2} .
$$

For any SPD matrix $A \in \mathbb{R}^{(n+1) \times(n+1)}$ and any vector $y \in \mathbb{R}^{n+1}$ let $\|y\|_{A}^{2}=y \cdot A y$ denote the associated norm. If $M:=M(K)$ denotes the local mass matrix for the $P_{1}$-conforming FEM from (6.4), the Cauchy-Schwarz inequality implies

$$
|x \cdot(0,1, \ldots, 1)| \leq\|(0,1, \ldots, 1)\|_{M^{-1}}\|x\|_{M}=\|(0,1, \ldots, 1)\|_{M^{-1}}\|f\|_{L^{2}(K)} .
$$

An elementary calculation with the Sherman-Morisson formula shows $\|(0,1, \ldots, 1)\|_{M^{-1}}^{2}=$ $2 n(n+1) /|K|$. The combination of this with the previous displayed formulas concludes the proof of the second inequality in (D1). 
In the above notation the Cauchy-Schwarz inequality implies

$$
\left|f\left(P_{0}\right)\right|=|x \cdot(1,0, \ldots, 0)| \leq\|(1,0, \ldots, 0)\|_{M^{-1}}\|f\|_{L^{2}(K)} .
$$

This and $\|(1,0, \ldots, 0)\|_{M^{-1}}^{2}=(n+1)^{2} /|K|$ prove the third inequality in (D1).

Since each side $F$ of a $n$-simplex is a $(n-1)$-simplex, the point estimate in Lemma $\mathrm{D}$ translates to sides; it coincides with the optimal estimate in Lemma A for $n=2$.

Corollary D. Let $F \in \mathcal{F}(K)$ be a side of a n-simplex $K \subset \mathbb{R}^{n}$ with vertex $P \in \mathcal{N}(F)$ and positive surface measure $|F|$, then any affine function $f \in P_{1}(F)$ satisfies

$$
|f(P)|^{2} \leq \frac{n^{2}}{|F|}\|f\|_{L^{2}(F)}^{2} .
$$

\section{References}

[1] D. Boffi, F. Brezzi and M. Fortin. Mixed finite element methods and applications. Springer Series in Computational Mathematics. Springer Berlin Heidelberg, 2013.

[2] D. Braess. Finite Elemente: Theorie, schnelle Löser und Anwendungen in der Elastizitätstheorie. Springer-Verlag, 2013.

[3] S. Brenner and R. Scott. The mathematical theory of finite element methods. Texts in Applied Mathematics. Springer, 2008.

[4] C. Carstensen, M. Feischl, M. Page and D. Praetorius. Axioms of adaptivity. Comput. Math. Appl., 67:6 (2014), 1195-1253.

[5] C. Carstensen and D. Gallistl. Guaranteed lower eigenvalue bounds for the biharmonic equation. Numer. Math., 126:1 (2014), 33-51.

[6] C. Carstensen, D. Gallistl and J. Hu. A discrete Helmholtz decomposition with Morley finite element functions and the optimality of adaptive finite element schemes. Comput. Math. Appl., 68:12 (2014), 2167-2181.

[7] C. Carstensen, D. Gallistl and M. Schedensack. Discrete reliability for Crouzeix-Raviart FEMs. SIAM J. Numer. Anal., 51:5 (2013), 2935-2955.

[8] C. Carstensen, D. Gallistl and M. Schedensack. Adaptive nonconforming Crouzeix-Raviart FEM for eigenvalue problems. Math. Comp., 84 (2015), 1061-1087.

[9] C. Carstensen and J. Gedicke. Guaranteed lower bounds for eigenvalues. Math. Comp., 83:290 (2014), 2605-2629.

[10] C. Carstensen and F. Hellwig. Constants in discrete Poincaré and Friedrichs inequalities and discrete quasi-interpolation. Comput. Methods Appl. Math., 18:3 (2017), 433-450.

[11] C. Carstensen and F. Hellwig. Optimal convergence rates for adaptive lowest-order discontinuous Petrov-Galerkin schemes. SIAM J. Numer. Anal., 56:2 (2018), 1091-1111.

[12] C. Carstensen, J. Hu and A. Orlando. Framework for the a posteriori error analysis of nonconforming finite element. SIAM J. Numer. Anal., 45:1 (2007), 68-82.

[13] C. Carstensen and K. Köhler. Nonconforming FEM for the obstacle problem. IMA J. Numer. Anal., 37:1 (2017), 64-93.

[14] C. Carstensen, D. Peterseim and M. Schedensack. Comparison results of finite element methods for the Poisson model problem. SIAM J. Numer. Anal., 50:6 (2012), 2803-2823.

[15] C. Carstensen and H. Rabus. Axioms of adaptivity with separate marking for data resolution. SIAM J. Numer. Anal., 55:6 (2017), 2644-2665.

[16] P.G. Ciarlet. The finite element method for elliptic problems, volume 4 of Studies in Mathematics and its Applications. North-Holland, Amsterdam, 1978. 
[17] M. Crouzeix and P.A. Raviart. Conforming and nonconforming finite element methods for solving the stationary Stokes equations I. ESAIM: Mathematical Modelling and Numerical Analysis Modélisation Mathématique et Analyse Numérique, 7:R3 (1973), 33-75.

[18] D. Gallistl. Morley finite element method for the eigenvalues of the biharmonic operator. IMA J. Numer. Anal., 35:4 (2015), 1779-1811.

[19] T. Gudi. A new error analysis for discontinuous finite element methods for linear elliptic problems. Math. Comp., 79:272 (2010), 2169-2189.

[20] J. Hu, Z. Shi and J. Xu. Convergence and optimality of the adaptive Morley element method. Numer. Math., 121:4 (2012), 731-752.

[21] W. Ming and J. Xu. The Morley element for fourth order elliptic equations in any dimensions. Numerische Mathematik, 103:1 (2006), 155-169.

[22] L.S.D. Morley. The triangular equilibrium element in the solution of plate bending problems. Aeronautical Quarterly, 19:2 (1968), 149-169.

[23] C. Ortner and D. Praetorius. On the convergence of adaptive nonconforming finite element methods for a class of convex variational problems. SIAM J. Numer. Anal., 49:1 (2011), 346-367.

[24] H. Rabus. A natural adaptive nonconforming FEM of quasi-optimal complexity. Comput. Methods Appl. Math., 10:3 (2010), 315-325.

[25] R. Stevenson. The completion of locally refined simplicial partitions created by bisection. Math. Comp., 77:261 (2008), 227-241.

[26] N. Walkington. A $C^{1}$ tetrahedral finite element without edge degrees of freedom. SIAM J. Numerical Analysis, 52:1 (2014), 330-342.

[27] S. Zhang. A family of 3D continuously differentiable finite elements on tetrahedral grids. Applied Numerical Mathematics, 59:1 (2009), 219-233. 